ARTICLE

https://doi.org/10.1038/s41467-019-11061-8

\title{
Structural insights into E1 recognition and the ubiquitin-conjugating activity of the E2 enzyme Cdc34
}

Katelyn M. Williams ${ }^{1}$, Shuo Qie ${ }^{1}$, James H. Atkison ${ }^{1}$, Sabrina Salazar-Arango ${ }^{1}$, J. Alan Diehl (1) ${ }^{1} \&$ Shaun K. Olsen (1) ${ }^{1}$

Ubiquitin (Ub) signaling requires the sequential interactions and activities of three enzymes, E1, E2, and E3. Cdc34 is an E2 that plays a key role in regulating cell cycle progression and requires unique structural elements to function. The molecular basis by which $\mathrm{Cdc} 34$ engages its E1 and the structural mechanisms by which its unique C-terminal extension functions in Cdc34 activity are unknown. Here, we present crystal structures of Cdc34 alone and in complex with E1, and a Cdc34 Ub thioester mimetic that represents the product of Uba1-Cdc34 Ub transthiolation. These structures reveal conformational changes in Uba1 and Cdc34 and a unique binding mode that are required for transthiolation. The Cdc34 Ub structure reveals contacts between the Cdc34 C-terminal extension and Ub that stabilize Cdc34 Ub in a closed conformation and are critical for Ub discharge. Altogether, our structural, biochemical, and cell-based studies provide insights into the molecular mechanisms by which Cdc34 function in cells.

\footnotetext{
${ }^{1}$ Department of Biochemistry \& Molecular Biology and Hollings Cancer Center, Medical University of South Carolina, Charleston, SC 29425, USA Correspondence and requests for materials should be addressed to S.K.O. (email: olsensk@musc.edu)
} 
$\mathrm{R}$ eversible post translational modification of proteins by ubiquitin (Ub) regulates nearly every aspect of eukaryotic biology. Cell cycle progression is among the best studied processes controlled by Ub signaling and began with the observation that $\mathrm{Cdc} 34$, an E2 ubiquitin-conjugating enzyme, is essential for cell cycle progression in yeast ${ }^{1,2}$. As an E2, Cdc34 is the central player in a cascade of enzymes that includes an E1 ubiquitin activating enzyme, Uba1, and a large family of SkpCullin-F-box (SCF) E3 ubiquitin ligases that function in tandem to assemble Lys48-linked Ub chains on proteins to target them for proteasomal degradation ${ }^{3-5}$. Specifically, Cdc34 is best known to regulate G1-S checkpoint progression by targeting cyclindependent kinase inhibitors such as Sic1/p27 for degradation ${ }^{6-9}$. More recently, Cdc34 has been implicated in human pathologies including elevated Cdc34 levels in several cancers ${ }^{10-14}$ and Cdc34 regulation of oncoproteins ${ }^{15,16}$. Accordingly, expression of a dominant-negative Cdc34 mutant enhances anti-myeloma activity of Bortezomib ${ }^{17}$ and cisplatin therapy inhibits Cdc34mediated degradation of ATF5, a prosurvival transcription factor $^{18}$, generating interest in the SCF-Cdc34 pathway as a potential therapeutic target ${ }^{19-21}$.

Ubal is a multidomain enzyme that serves as the gatekeeper of the Ub conjugation cascade by activating Ub in a two-step process involving adenylation and thioester bond formation followed by transfer of Ub to E2s in a process termed E1-E2 thioester transfer or transthiolation $3,22,23$. Each Ubal domain plays a distinct functional role with active and inactive adenylation domains (AAD and IAD) that adenylate the C-terminus of $\mathrm{Ub}^{24-27}$; a Cys domain (split into first and second catalytic cysteine half domains, $\mathrm{SCCH}$ and $\mathrm{FCCH}^{28}$ ) that harbors the catalytic cysteine for thioester bond formation with $\mathrm{Ub}$; and a ubiquitin fold domain (UFD) that is involved in molecular recognition of $\mathrm{E} 2 \mathrm{~s}^{25,29,30}$. Cdc34 is one of tens of E2s that must function with Uba1 despite significant differences at their predicted UFDinteracting surfaces. For example, Cdc34 lacks a conserved basic motif known to be important for E1-E2 thioester transfer for other $\mathrm{E} 2 \mathrm{~s}^{31}$; has an $\mathrm{N}$-terminal extension proximal to the major UFD-binding region of E2s; and harbors amino acids with very different physiochemical properties at predicted E1-binding positions. Thus, the Uba1-Cdc34 complex is likely to utilize a distinct binding mode compared to previously characterized Uba1-E2 complexes ${ }^{32,33}$ and, therefore, may provide insights into the molecular rules governing promiscuity in Uba1-E2 interactions.

After E1-E2 thioester transfer, the resulting Cdc34 Ub thioester intermediate (Cdc34 Ub, where $\sim$ indicates a thioester) is recruited to SCF E3 ubiquitin ligases that catalyze extension of Lys48-linked polyUb chains. SCF ligases are multidomain enzymes that include Rbx1, a RING-domain protein that harbors the catalytic activity and engages E2 $\mathrm{Ub}^{34-37}$. E2 Ub adopts an array of conformations ${ }^{38-42}$ and one catalytic mechanism whereby RING stabilizes E2 Ub in a closed conformation is established $^{43-45}$, though this has not been confirmed for Rbx1/ Cdc34. Additionally, Cdc34 contains unique structural features that are crucial for efficient polyUb chain extension through poorly understood mechanisms, including an acidic loop insertion proximal to the active site and a long C-terminal extension. The C-terminal extension has two regions with distinct biological functions: the distal highly acidic region binds a basic canyon on SCF component Cul1 for rapid Cdc34 Ub/SCF complex formation ${ }^{46,47}$ and the region immediately proximal to the Ubc core is implicated in self-association and non-covalent interaction with $\mathrm{Ub}$ thioester, $\mathrm{Ub}(\mathrm{t})^{48-50}$. Previously resolved structures of $\mathrm{Cdc} 34^{19,51}$, including one with $\mathrm{Ub}$, utilized constructs that were truncated just beyond the Ubc core due to presumed disordering of the tail ${ }^{49}$, making analysis of interactions between the tail and
$\mathrm{Ub}(\mathrm{t})$ impossible. Thus, despite work spanning several decades, there are still many questions about the mechanistic roles of this C-terminal extension.

To better understand the molecular bases of Cdc34 activities, we here determine crystal structures of Cdc34 alone and in complex with Uba1, as well as a Cdc34 Ub mimetic that represents the product of E1-E2 thioester transfer. The structures illuminate conformational changes in both Cdc34 and Uba1 that are required for complex formation and reveal a unique binding mode compared to previously characterized E1-E2 complexes. Further, the Cdc34 Ub mimetic structure reveals that the proximal C-terminal extension engages $\mathrm{Ub}(\mathrm{t})$ and stabilizes $\mathrm{Cdc} 34 \sim \mathrm{Ub}$ in a closed conformation. Further, these contacts are critical for Ub discharge in vitro and in cells. Collectively, our structural, biochemical, and cell-based studies contribute insights to the molecular mechanisms of Cdc34 activity in the cell.

\section{Results}

Overall architecture of a ScUba1-Cdc34 ${ }^{\Delta \text { dist }} / \mathrm{Ub}$ (a) complex. A number of sequence and structural features unique to Cdc34 suggest that it engages Ubal via a distinct binding mode during E1-E2 thioester transfer compared to other E2s. First, the physiochemical properties of amino acids in Cdc34 predicted to interact with the Uba1 UFD differ significantly compared to other E2s, including the absence of a highly conserved basic motif that has been shown to be critical for E1-E2 thioester transfer ${ }^{31}$. Cdc34 also harbors a short N-terminal extension proximal to the major UFD-binding region of E2s that is in position to make contacts to Uba1 that are specific to Cdc34. To reveal the roles of these unique Cdc34 features in Ubal binding, a crystal structure of a ternary $S$. cerevisiae Uba1-Cdc34/Ub/Mg•ATP complex (Fig. 1a and Supplementary Table 1), hereafter referred to as Uba1-Cdc34, was determined using a previously described crosslinking strategy that stabilizes the complex via a disulfide bond between the Ubal and Cdc34 catalytic cysteines ${ }^{32}$, in vitro E1-E2 thioester transfer assays demonstrate that both CTDprox (residues 171-195) and CTD ${ }^{\text {dist }}$ (residues 196-295) of the extended Cdc34 C-terminus are dispensable for thioester transfer activity (Fig. 1c and Supplementary Fig. 1) and the best diffracting crystals were obtained with a Cdc34 construct lacking CTD ${ }^{\text {dist }}\left(\mathrm{Cdc} 4^{\Delta \text { dist }}\right)$. Of note, all bar graph representations of biochemical data in these studies represent quantification of product formation at the indicated timepoint, not the kinetic rate of product formation. The Uba1-Cdc34 structure was determined to $2.07 \AA$ resolution (Supplementary Table 1) with two nearly identical copies of the complex in the crystallographic asymmetric unit.

Globally, the Uba1-Cdc34 complex exhibits a conserved architecture with the IAD and $\mathrm{AAD}$ engaging the $\mathrm{Ub}$ adenylate, $\mathrm{Ub}(\mathrm{a})$, and with $\mathrm{Cdc} 34$ sandwiched between the UFD and SCCH of Uba1 (Fig. 1b). Available structures of Uba1 indicate a range of conformations for the UFD that span from distal to proximal relative to the $\mathrm{SCCH}^{25,32,52,53}$. Previous studies suggest that the Uba1 UFD initially recruits E2 in a distal conformation and subsequently rotates into a proximal conformation to place the E2 catalytic cysteine near the Uba1 Ub thioester bond to allow transfer of Ub from Uba1 to E2. Indeed, when Cdc34 $4^{\mathrm{E} 1 \text {-bound }}$ is docked onto the distal UFD of Ubal ${ }^{\text {apo }}$ (PDB: $\left.3 C M M\right)^{52}$, the catalytic cysteines are separated by $13 \AA$ and would be unable to participate in thioester transfer (Fig. 1d, left). Comparison of Uba1 Cdc34-bound to Uba $1^{\text {apo }}$ reveals a $20^{\circ}$ rotation of the UFD proximally (Fig. 1d, middle) to bring the catalytic cysteines of Ubal and Cdc34 together for Ub thioester transfer (Fig. 1d, right).

Molecular recognition of Cdc34 by Uba1. Analysis of the Uba1Cdc34 structure reveals three networks of contacts at distinct 
a S. cerevisiae Uba1-Cdc34 $4^{\text {dist }} / \mathrm{Ub}(\mathrm{a})$
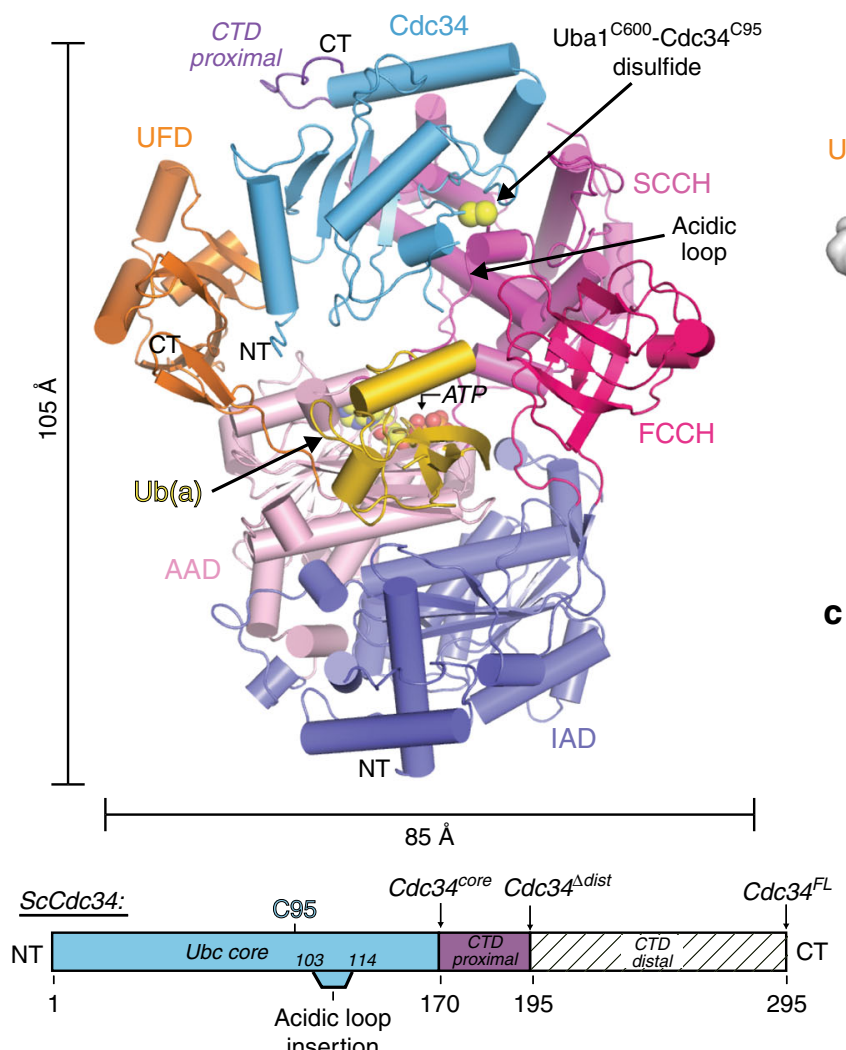

b

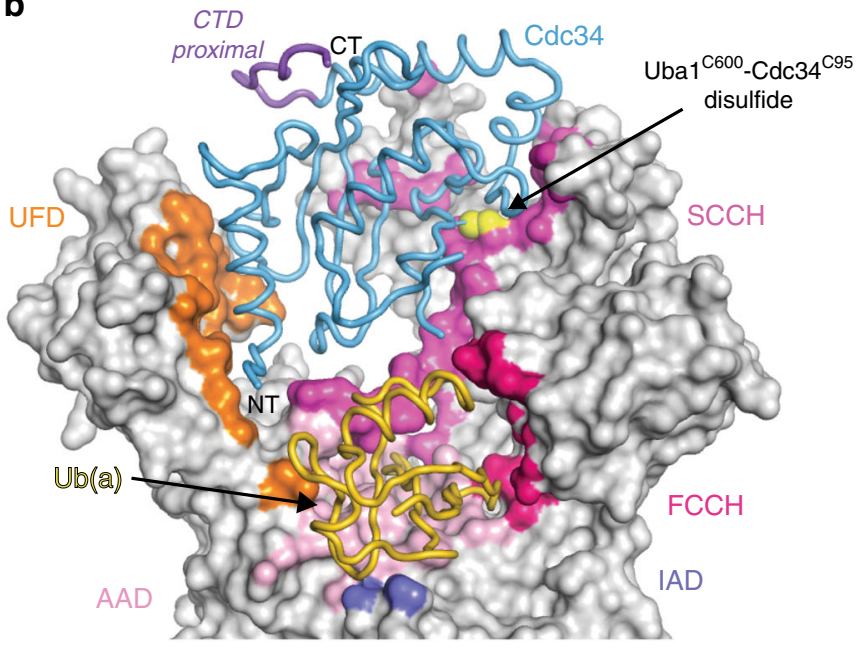

C E1-E2 Ub thioester transfer assays:

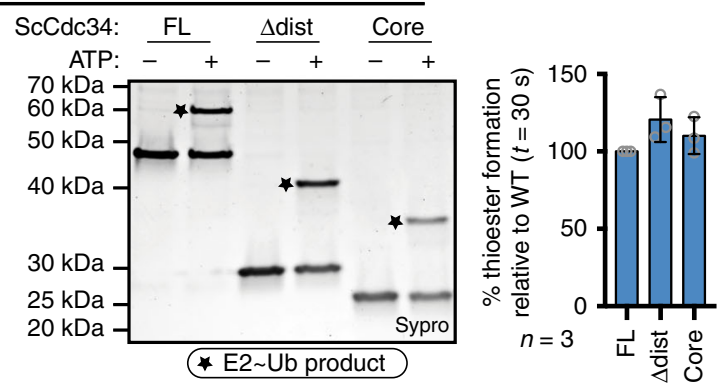

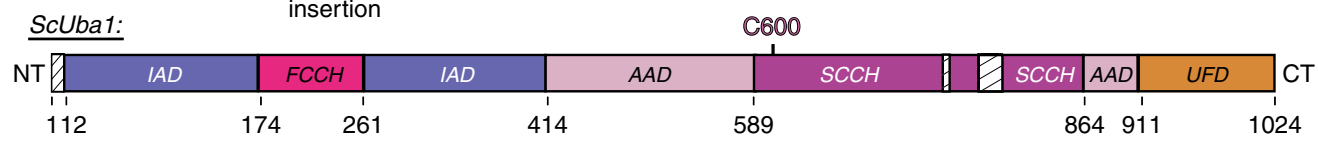

d S. cerevisiae Uba1-Cdc34 (UFD dist)

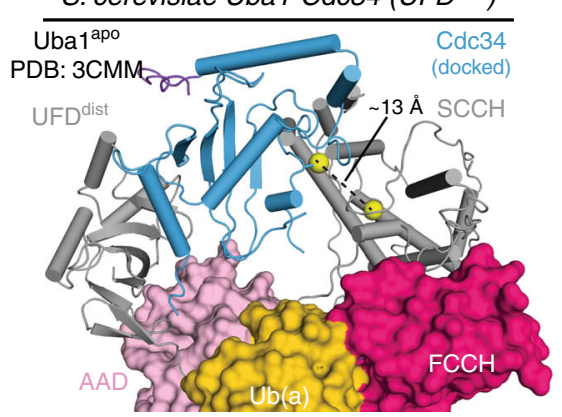

S. cerevisiae Uba $1^{a p o}$ vs. Uba ${ }^{\text {Cdc } 34}$

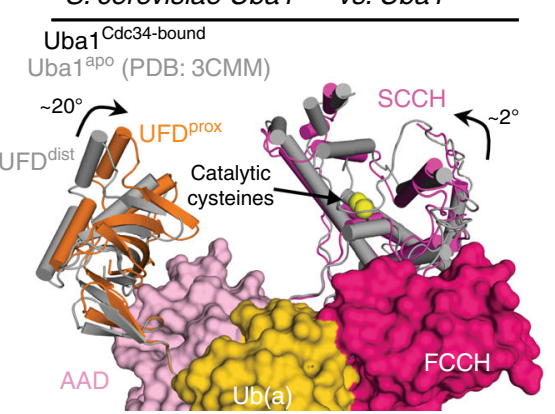

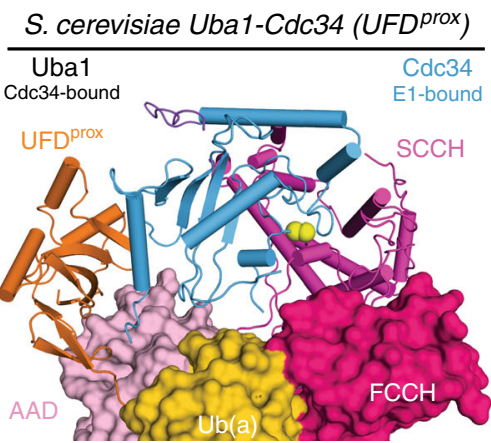

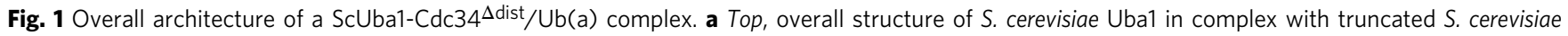
Cdc34, Cdc34 ${ }^{\text {dist }}$ (blue), and ubiquitin adenylate, Ub(a) (gold), with Uba1 domains colored and labeled. Catalytic cysteines are indicated by yellow spheres. Bottom, schematic representations of the ScUba1 and ScCdc34 constructs with domains colored as above. b Uba1-Cdc34 structure with Uba1 surface representation in gray with only contacting residues colored. Cdc34 ${ }^{\text {dist }}$ and Ub(a) are represented as worms. c E1-E2 thioester transfer assays for $\mathrm{Cdc} 34$ variants with boundaries as indicated in schematic, and with normalized quantification of E2 thioester formed represented as mean \pm SD with three independent replicates shown as gray circles, right. Stars indicate the E2 Ub thioester product. Source data are provided as a Source Data file. $\mathbf{d}$ Left, Cdc34 ${ }^{\mathrm{E1} \text {-bound }}$ modeled onto Uba1 ${ }^{\mathrm{apo}}$ UFD (gray) in open conformation (PDB:3CMM); distance between catalytic cysteines is indicated by a dashed line. middle, ScUba1Cdc34-bound/Ub(a) as in a with ScUba1apo/Ub(a) superimposed in gray. Arrows indicate rotation of the UFD and SCCH when bound to Cdc34. Right, ScUba1-Cdc34 $4_{\text {dist }} / \mathrm{Ub}(\mathrm{a})$ complex structure colored as in a

interfaces: Cdc34/UFD, Cdc34/SCCH, and a tripartite interaction between $\mathrm{Cdc} 34, \mathrm{Ub}(\mathrm{a})$, and the Ubal crossover loop that connects the AAD to the SCCH (Fig. 2a). One of the most prominent features of the UFD is a conserved acidic patch that includes Glu1004, Asp1008, Asp1014, and Glu1016. At the heart of the Cdc34/UFD interface lies a network of salt bridges between the UFD acidic patch and basic residues of Cdc34 (Fig. 2d).
Specifically, Arg14 and Arg17 of Cdc34 helix A (hA) form salt bridges with Asp1014 and Glu1016 on the UFD, as well as a water-mediated hydrogen bond with Asp1008 (Supplementary Fig. 2). Introduction of charge reversal mutations at any of these positions on Uba1 or Cdc34 results in a significant loss of thioester transfer activity (Fig. 2e). Moreover, combining Cdc34 (R14E/R17E) and Ubal (D1014R/E1016R) charge reversal 


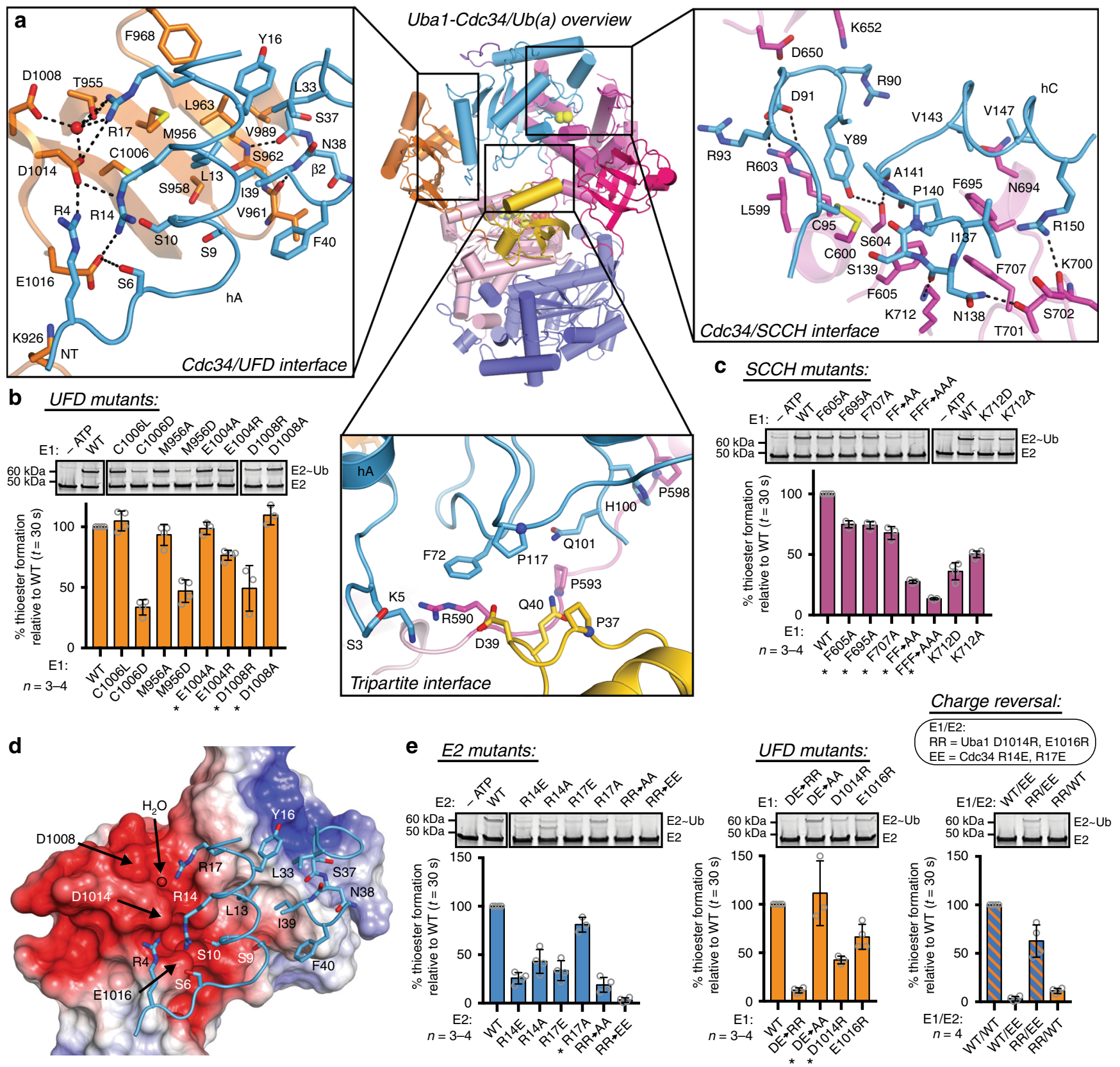

Fig. 2 Combinatorial recognition of Cdc34 $4^{\Delta \text { dist }}$ by ScUba1. a Top middle, overall structure of Uba1-Cdc34 $4^{\Delta \text { dist} / U b ~ a s ~ i n ~ F i g . ~ 1 a . ~ T o p ~ l e f t, ~ n e t w o r k ~ o f ~ c o n t a c t s ~}$ between Uba1 UFD and Cdc34 with involved residues shown as sticks with red oxygen atoms, blue nitrogen atoms, and yellow sulfur atoms. Hydrogen bonds are indicated by dashed lines. Top right, interaction network between $\mathrm{Cdc} 34$ and Uba1 SCCH. Bottom middle, tripartite network between $\mathrm{Cdc} 34$, Ub (a), and Uba1 crossover loop. b, c E1-E2 thioester transfer assays of the indicated mutants for Uba1 UFD (b) and SCCH (c). Data are represented by mean \pm SD with representative replicates labeled above and individual replicates shown as gray circles. Biochemical assays have four independent replicates, unless indicated by an asterisk when there are three replicates. $\mathbf{d}$ Electrostatic surface of Uba1 UFD with Cdc34 contacting residues overlaid as in a. e E1-E2 thioester transfer assays of the indicated mutants for Cdc34 (E2), Uba1 UFD, and a charge reversal rescue assay with mutant Uba1 and Cdc34, represented as in $\mathbf{b}$, c. Source data are provided as a Source Data file

mutants rescues a near complete loss of activity when either mutant was tested with a WT partner (Fig. 2e). This confirms the critical importance of these salt bridge interactions in the proper positioning of Cdc34 for E1-E2 thioester transfer. Aligning with these findings, previous studies have shown a reduction in $S$. cerevisiae viability when Arg14, Arg17, and Glu18 are all mutated to $\mathrm{Ala}^{54}$.

At the periphery of the interactions described above is a supporting network of contacts between the UFD and Cdc34 hA that includes UFD residues Lys926, Thr955, Met956, Leu963,
Phe968, Cys1006, and Val1015; and hA residues Ser6, Ser10, Leu13, and Tyr16. Ser3 and Arg4 from the Cdc34 N-terminal extension also reach up to contact Asp1014, Val1015, and Glu1016 (Fig. 2a). Additionally, the Ubal $\beta 29$-strand residues Val961 and Leu963 form backbone hydrogen bonds with $\beta 1-\beta 2$ loop and $\beta 2$-strand residues Ser37 and Ile39. Charge mutation of the supporting Uba1 residues Met956, Glu1004, Cys1006, and Asp1008 reduces efficiency of thioester transfer while more conservative Ala substitutions exhibit wild-type activity (Fig. 2b). This pattern suggests that, while these residues are buried at the 
interface of hA and the UFD, they are not individually critical for optimal positioning of Cdc34 for E1-E2 thioester transfer.

On the other side of $\mathrm{Cdc} 34$, the helix C/helix $\mathrm{D}(\mathrm{hC} / \mathrm{hD})$ surface forms an extensive network of contacts with the SCCH around the catalytic cysteines (Fig. 2a, right). Proximal to the active site cysteines, Cdc34 Tyr89 contacts Uba1 Leu599, Cys600, Arg603, and Ser604, including a hydrogen bond to Ser604 (Fig. 2a, right). Additionally, Cdc34 Ser139 and Ala141 contact Cys600 and Ser604. To the left of the catalytic cysteines, Cdc34 Asp91 forms a salt bridge with Arg603 of Uba1. To the right of the catalytic cysteines, Cdc34 Ile137 contacts Uba1 Ser702, and Asn138 creates a network of contacts with Thr701 and Lys712, including two hydrogen bonds. Highlighting its importance at this interface, disruption of the Lys712 hydrogen bond by mutation to Asp or Ala results in a moderate loss of thioester transfer activity (Fig. 2c). Cdc34 Pro140 inserts into a hydrophobic pocket on Ubal formed by three phenylalanine residues 605,695 , and 707, mutation of which results in a loss of E1-E2 thioester transfer activity (Fig. 2c). Finally, a tripartite network of contacts between $\mathrm{Cdc} 34, \mathrm{Ub}(\mathrm{a})$, and the Ubal crossover loop is observed wherein Cdc34 Phe72 contacts Uba1 Arg590; Ub Gln40 contacts Uba1 Pro593; and Ub Asp39 contacts Uba1 Arg590 and Cdc34 Phe72 (Fig. 2a, bottom middle). Thus, Cdc34 and Uba1 engage in extensive contacts at three distinct interfaces for productive complex formation and transthiolation.

A flexible Ubal architecture accommodates E2-binding modes. Comparison of the Uba1-Cdc34 complex to previously determined Uba1-Ubc4 and Uba1-Ubc15 structures ${ }^{32,33}$ reveals three distinct Uba1-binding modes. Superimposition of the UFDs of these structures unmasks significant differences in how hA of each E2 engages the UFD (Fig. 3a, middle). Specifically, hA of Cdc 34 rotates $7^{\circ}$ and translates $0.9 \AA$ toward the bottom of the UFD with respect to Ubc4. With respect to Ubc15, hA of Cdc34 rotates $13^{\circ}$ and translates $1 \AA$ toward the top of the UFD (Fig. 3a, left; $3 b)$. These seemingly subtle differences at the hA/UFD interface result in significantly different positioning of the active sites and SCCH-binding surfaces of the E2s relative to the UFD (Fig. 3a, middle). Highlighting this, the catalytic cysteine of Cdc34 has translated $3.7 \AA$ compared to Ubc 4 and $6.9 \AA$ compared to Ubc15 (Fig. 3a, right). In contrast, superimposition by the SCCH reveals that the $\mathrm{E} 2 / \mathrm{SCCH}$ interface is better conserved than the $\mathrm{UFD} / \mathrm{E} 2$ interface (Fig. 3c). The overall orientation of the E2s relative to the $\mathrm{SCCH}$ are similar and the catalytic cysteines remain in proximity via a common network of contacts (Supplementary Fig. 3).

A variable E2/UFD interface that results in positioning of E2 catalytic cysteines up to $7 \AA$ apart from each other (Fig. 3a, b) together with a conserved E2/SCCH interface (Fig. 3c) raises the question of how Uba1 and E2 active sites can come together as required for E1-E2 thioester transfer. Superimposition by the Ubal adenylation domains, which are the rigid body of the enzyme ${ }^{52}$, reveals that the architecture of Ubal in each of the structures is distinct, with the UFD and SCCH exhibiting relative differences in rotation of $5-9^{\circ}$ and $4-6^{\circ}$, respectively (Fig. $3 \mathrm{~d}$ ). Altogether, these distinct domain rotations are the mechanism by which E1 and E2 active sites are brought into proximity during E1-E2 thioester transfer despite differences in E2/UFDbinding mode.

Structural plasticity at the E2/UFD interface. Comparison of the Uba1-Cdc34, Uba1-Ubc4, and Uba1-Ubc15 E2/UFD interfaces reveals distinct networks of contacts (Fig. 4a, top) that reflect the markedly different angles at which E2 hAs engage the UFD (Fig. 3a). One clear difference at this interface is the network of interactions mediated by the Ubal UFD acidic patch. Previous modeling and biochemical experiments led to the prediction that the acidic patch interacts with a highly conserved three residue basic motif on E2 hA that corresponds to ${ }^{4} \mathrm{KRINR}^{8}$ in $\mathrm{Ubc}^{31,52}$. Interestingly, although Cdc34 contains only the last basic residue in the motif, Arg14, Cdc34 interacts with a larger surface of the acidic patch than both Ubc4 and Ubc15 (Fig. 4a, middle). This is due to an additional basic residue proximal to Arg14, Arg17, which, facilitated by a distinct hA orientation, leads to extensive contacts between Arg14/Arg17 and the top of the UFD acidic patch that are critical for proper Uba1-Cdc34 thioester transfer in vitro (Fig. 2d, e) and Cdc34 activity in cells ${ }^{54}$. In contrast, Ubc4 lacks a basic residue equivalent to Arg17 and the orientation of $\mathrm{hA}$ is such that the top of the UFD acidic patch makes no contacts to Ubc4 (ref. ${ }^{32}$ ) (Fig. $4 \mathrm{a}$, top). Ubc15 also contains only the last basic residue of the basic motif, Lys12, and Lys15 that corresponds to Cdc34 Arg17. Contrary to the short-range salt bridge interactions between the acidic patch and well-ordered Cdc34 Arg14/Arg17, Ubc15 Lys12, and Lys15 are poorly ordered and participate in longer-range electrostatic interactions with the acidic patch (Fig. 4a, top). This is due to both the shorter lysine side chains and the presence of an acidic residue, Glu7, that necessitates a downward shift of Ubc15 hA to avoid electrostatic repulsion between Glu7 and the UFD acidic patch, positioning Lys12 and Lys15 out-of-range for potential salt bridge interactions (Fig. 4a, top).

To further probe the plasticity of the UFD/E2 interface, residues in Ubc4 and Ubc15 were mutagenized to more closely resemble Cdc34 (Fig. 4b and Supplementary Fig. 4). In Ubc4, mutating Lys4 to Cdc34 Ser10 resulted in a 2-fold loss of activity, while mutation of Ala11 to Cdc34 Arg17 had no significant effect on thioester transfer activity (Fig. $4 \mathrm{~b}$ and Supplementary Fig. 4). Interestingly, the double mutation $(\mathrm{KA} \rightarrow \mathrm{SR}$ ) rescued $\mathrm{K} 4 \mathrm{~S}$ activity loss, suggesting that $\mathrm{Ubc} 4$ may be able to interact with the acidic patch similarly to Cdc34. In Ubc15 a K12A mutant exhibits a 5fold loss of activity whereas K15A has a mild increase in activity (Fig. 4b and Supplementary Fig. 4). Surprisingly, a K12A/K15A double mutation results in a near complete loss of Ubc15 activity indicating that, while it is otherwise dispensable, Lys 15 becomes necessary in the absence of Lys12. This suggests that the UFD is able to compensate for loss of important E2 contacts by reorganizing to form favorable contacts with other residues in proximity, further supporting structural plasticity at the UFD/E2 interface as the mechanism underlying promiscuity in E1-E2 interactions.

Outside of the UFD acidic patch, a conserved set of UFD residues including Met956 and Cys1006 engage different sets of $\mathrm{E} 2 \mathrm{hA}$ residues due to the different orientations of $\mathrm{E} 2 \mathrm{hA}$ and sequence diversity among E2s at this region (Figs. 3a and 4). Another major region of variability at the E2/UFD interface involves the variable $\mathrm{N}$-terminal extensions of E2s (Fig. 4a). While Cdc34's extension projects outward between the UFD and AAD making additional contacts to the UFD acidic patch and AAD, the Ubc15 N-terminal extension takes a more downward path into the AAD, contacting a region of the UFD below the acidic patch and making far more contacts to the $\mathrm{AAD}^{33}$ (Fig. 4a). Conversely, Ubc4 does not possess an N-terminal extension and this is highlighted by fewer contacts to the UFD and no contacts to the AAD (Fig. 4a). More than 20 active human E2s contain variable N-terminal extensions, and this analysis suggests they may engage the $\mathrm{E} 1$ in distinct ways.

In summary, these analyses reveal that the Uba1 UFD utilizes a conserved core of residues to build distinct networks of contacts with divergent E2 hA sequences. Additionally, variable regions outside of this conserved UFD core, including the AAD, are engaged to accommodate unique $\mathrm{N}$-terminal extensions. 
a Uba1-E2 complexes - UFD superimposed
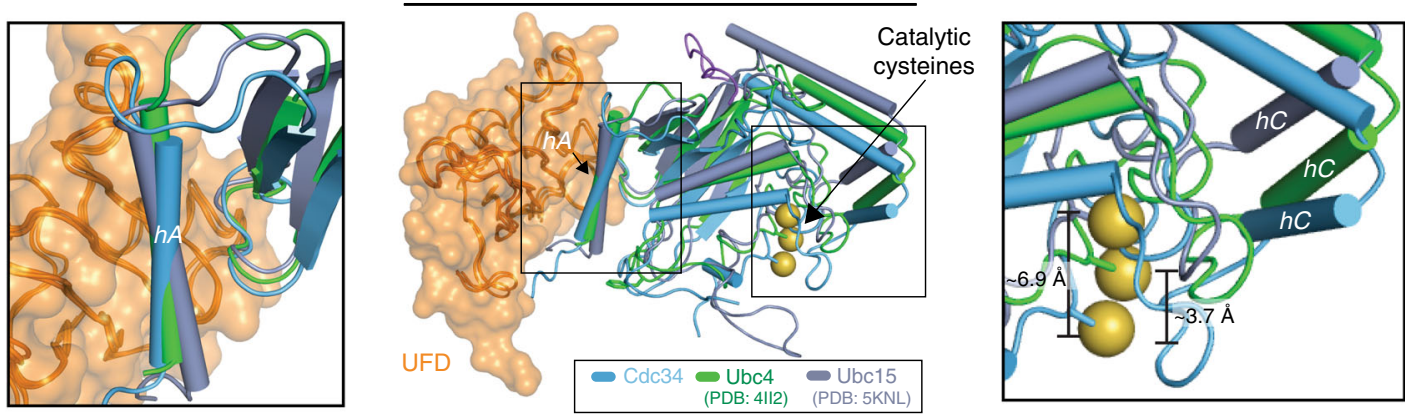

b

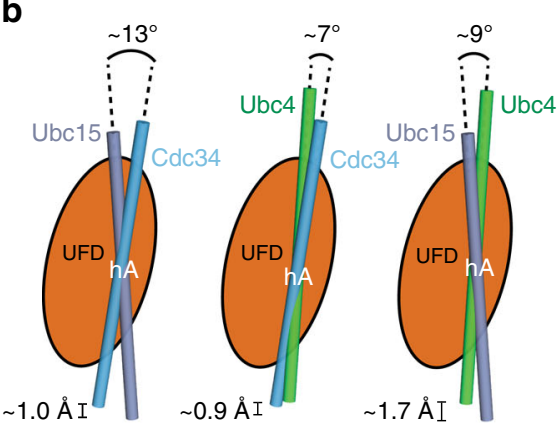

C Uba1-E2 complexes - SCCH superimposed

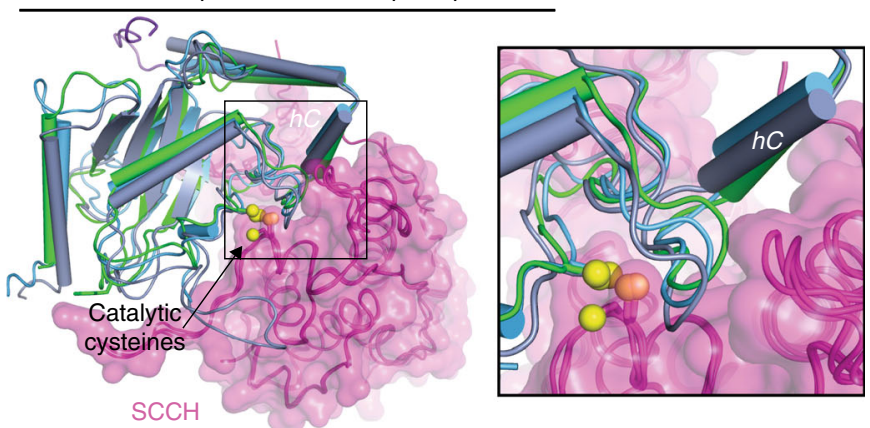

d Uba1-E2 complexes - $A A D$ superimposed

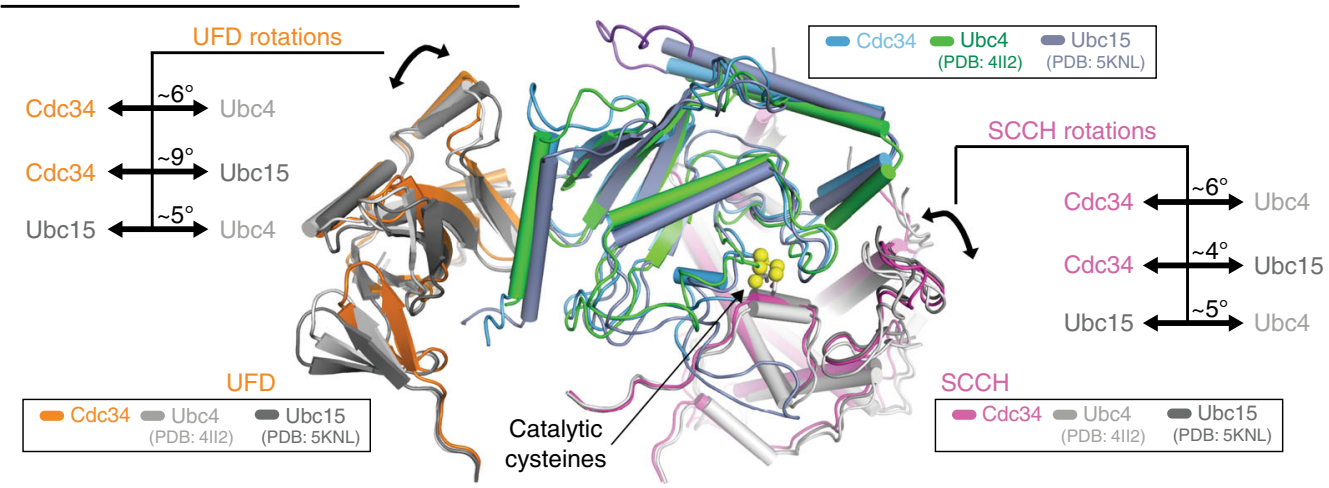

Fig. 3 Distinct Uba1 architectures accommodate different E2-binding modes. a Middle, superimposition of Uba1-Cdc34, Uba1-Ubc4 (PDB:4II2), and Uba1Ubc15 (PDB:5KNL) complexes by the UFD. Cdc34 is colored blue, Ubc4 is green, Ubc15 is gray. hA/UFD interface and catalytic cysteine positioning are highlighted by black boxes. Left, zoomed in, head-on view of E2 hA binding to Uba1 UFD. Right, zoomed in view of catalytic cysteine positions. b Cartoon schematic of difference in angle of hA bound to Uba1 for each E2 pair, as in $\mathbf{a}$. c Left, superimposition of Uba1-E2 complexes by the SCCH with the E2/ SCCH interface highlighted by a black box. Right, zoomed in view of the E2/SCCH interface. $\mathbf{d}$ Superimposition of Uba1-E2 complexes by the AAD with the E2, UFD, and SCCH shown. Relative differences in rotation for the UFD (left) and SCCH (right) are indicated by arrows

Altogether, this results in an altered orientation of each E2 hA relative to the UFD and necessitates distinct rotations of both the UFD and SCCH in order to bring E1 and E2 catalytic cysteines in proximity for productive thioester transfer in the context of different E1-E2-binding modes.

Cdc34 conformational changes accompany Uba1 binding. To investigate potential $\mathrm{Cdc} 34$ conformational changes that accompany Ubal binding, a $1.7 \AA$ structure of $\mathrm{ScCdc} 34^{\Delta \text { dist }}$ alone, Cdc34 $4^{\text {apo }}$, was determined (Fig. 5a and Supplementary Table 1). Comparison of $\mathrm{Cdc} 34^{\text {apo }}$ and $\mathrm{Cdc} 34^{\mathrm{E} 1 \text {-bound }}$ reveals two conformational changes that accompany Uba1 binding, both within E1-interacting regions. First, compared to Cdc $34^{\text {E1-bound, }}$ hA of $\mathrm{Cdc} 34^{\text {apo }}\left(\mathrm{hA}^{\mathrm{apo}}\right)$ is extended N-terminally by 5 residues, beginning at Ser3 instead of Ala8. When bound to Uba1, this extended region of hA melts but remains ordered and projects away from the helix (Figs. 2a, left, and 5a; and Supplementary Fig. 5). The second conformational change occurs on the opposite side of the $\mathrm{E} 2$, where $\mathrm{hC}$ of $\mathrm{Cdc} 34^{\text {apo }}\left(\mathrm{hC}^{\mathrm{apo}}\right)$ is extended $\mathrm{N}$ terminally by one residue and sits at a different angle compared to hCE1-bound (Fig. 5a and Supplementary Fig. 5). Modeling Cdc34 ${ }^{\text {apo }}$ onto the Uba1-Cdc34 complex reveals a mechanistic role for $\mathrm{Cdc} 34$ conformational changes that accompany Uba1 binding (Fig. 5b, middle). First, the extended $\mathrm{hA}^{\text {apo }}$ clashes with Uba1 AAD, including backbone and sidechain atoms of Ser3, Arg4, Lys5, and Ser6, whereas the melted region of $\mathrm{hA}^{\mathrm{E} 1-\text { bound }}$ packs into a small pocket between the UFD and AAD and makes additional contacts to Ubal (Fig. 5b, left). Second, the extension and altered angle of $\mathrm{hC}^{\mathrm{apo}}$ positions Val143 such that it clashes with the $\mathrm{SCCH}$, whereas $\mathrm{Cdc} 34^{\mathrm{E} 1 \text {-bound }}$ Val143 is shifted away from the $\mathrm{SCCH}$ and makes productive contacts with Ubal 
a
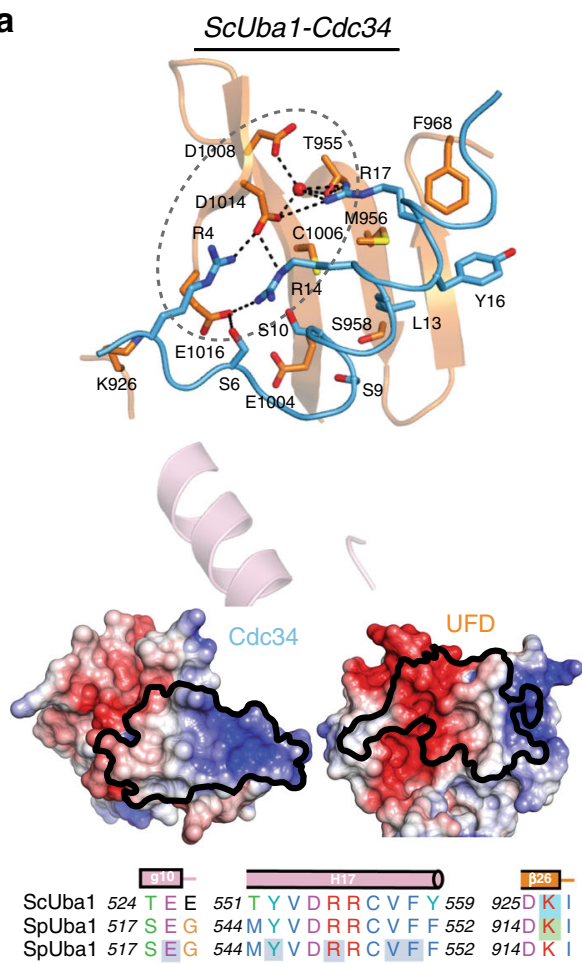
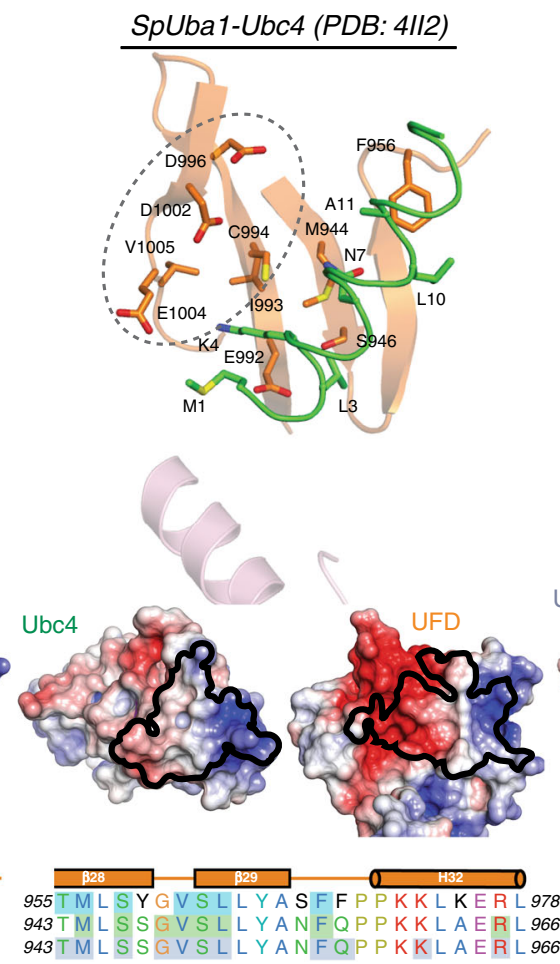

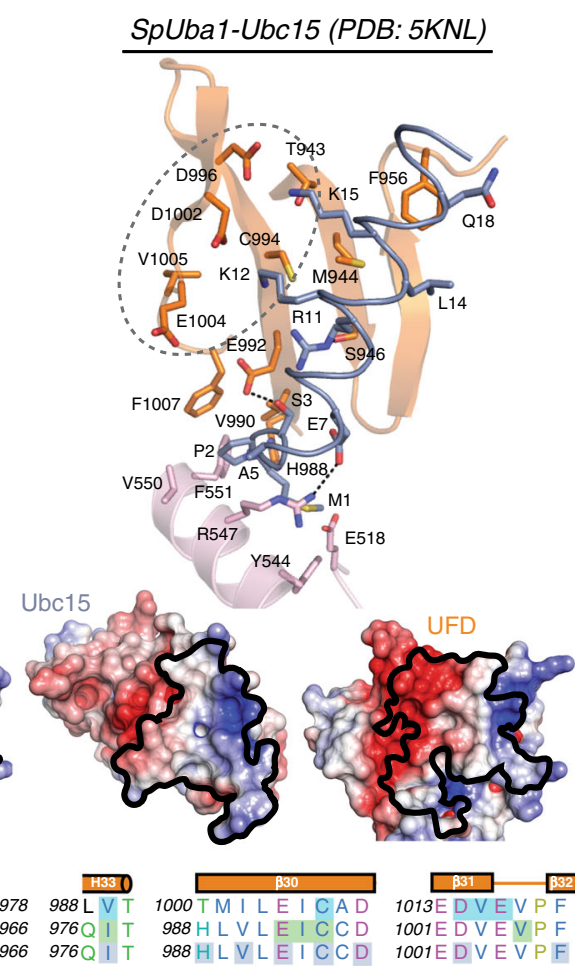

b

\section{Thioester transfer activity of Ubc4/Ubc15 "Cdc34" mutants}
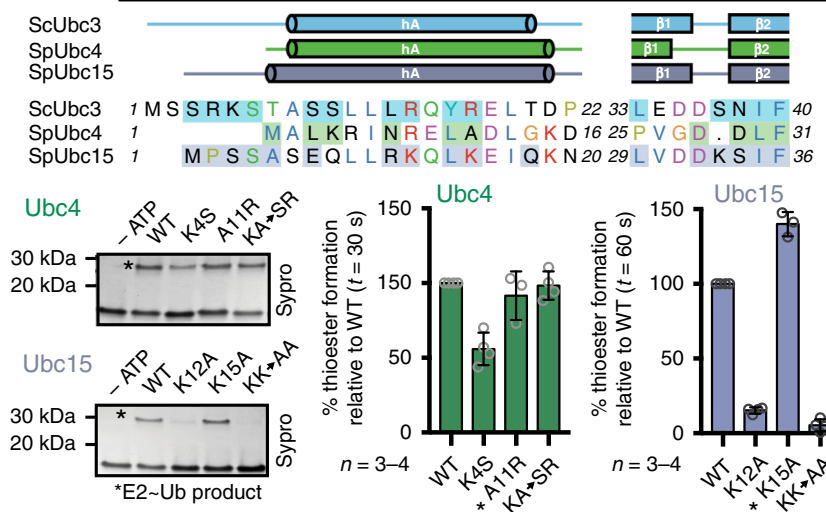

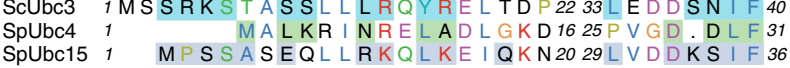
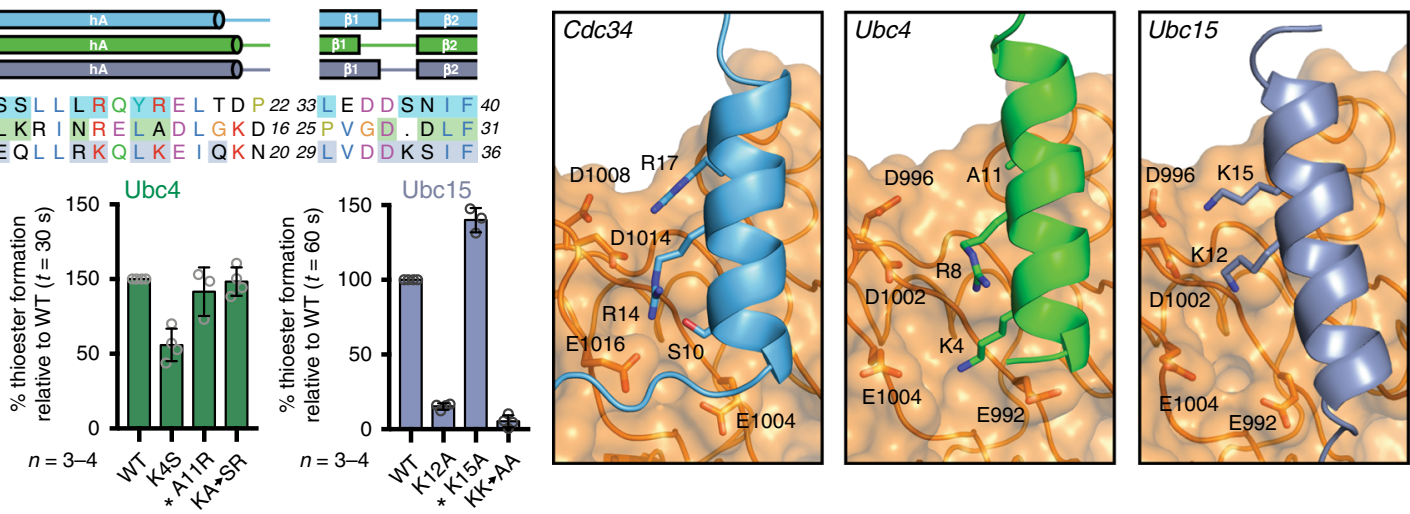

Fig. 4 The E1/Cdc34-binding mode is achieved via a unique network of contacts. a Top, interface between E2 and Uba1 UFD with involved residues shown as sticks for Cdc34 (left), Ubc4 (middle), and Ubc15 (right); colored as in Fig. 3. Dashed circles indicate UFD acidic patch binding region. Middle, open-book electrostatic surfaces of the E2/UFD interface with UFD footprint mapped onto E2 (left) and E2 footprint mapped onto UFD (right) for each E2 above. Bottom, sequence alignment of ScUba1 and SpUba1 AAD and UFD with corresponding secondary structure cartoon shown above sequence. Shaded boxes indicate Uba1 residues that interact with the corresponding E2: Cdc34 (blue), Ubc4 (green), and Ubc15 (gray). b Top left, sequence alignment of E2 UFD/ AAD-interacting region with secondary structure cartoon and interacting residues shaded as in $\mathbf{a}$. Bottom left, E1-E2 thioester transfer assays of the indicated mutants for Ubc4 and Ubc15. Data are represented by mean \pm SD with representative replicates labeled to the left and individual replicates shown as gray circles. Biochemical assays have four independent replicates, unless indicated by an asterisk when there are three replicates. Source data are provided as a Source Data file. Right, interface between UFD acidic patch and E2 hA residues corresponding to Cdc34 Arg14/Arg17

Asn694 and Phe695 (Figs. 2a, right; and 5b, right). Thus, these findings suggest that the observed Cdc34 conformational changes that accompany Ubal binding are important for productive Uba1-Cdc34 complex formation.

The Cdc34 CTDprox extension is involved in Ub discharge. The Cdc34 C-terminal extension is required for efficient Cdc34 function with CTD prox and CTD ${ }^{\text {dist }}$ playing distinct roles ${ }^{46-50,55}$. While a role for $\mathrm{CTD}^{\text {dist }}$ in facilitating rapid association of the Cdc34 Ub/SCF complex by binding to a basic canyon on Cull has been demonstrated $46,47,56$, the molecular basis by which
CTD prox promotes Cdc34 activity is less clear. Although nuclear magnetic resonance studies suggest interactions between CTD prox and $\mathrm{Ub}(\mathrm{t})^{49,50}$, the structural basis for the interaction and the mechanism for increased Cdc34 activity are unknown. Analysis of our $\mathrm{Cdc} 34^{\text {apo }}$ and $\mathrm{Cdc} 34^{\mathrm{E} 1 \text {-bound }}$ structures reveals additional ordering of CTDprox beyond what has been observed in previously determined Cdc34 structures ${ }^{19,51}$ (Fig. 5a, c). This additional ordered CTD ${ }^{\text {rox }}$ region engages in extensive contacts with the backside of Cdc34 and projects toward the anticipated location of $\mathrm{Ub}(\mathrm{t})$ in the closed conformation (Fig. 5c). These findings along with evidence for CTD prox participation in non-covalent contacts to $\mathrm{Ub}(\mathrm{t})^{49,50}$ led us to hypothesize that CTDprox extends 
a Cdc34 hA changes upon Uba1-binding

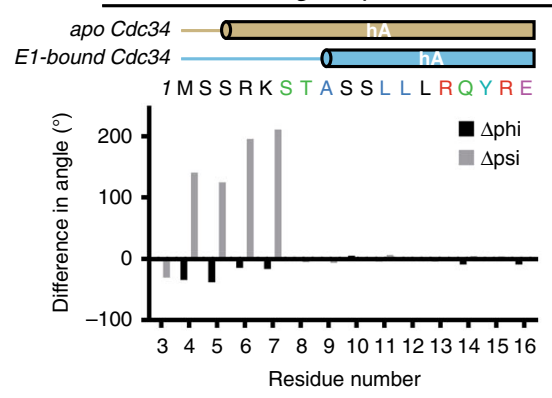

b

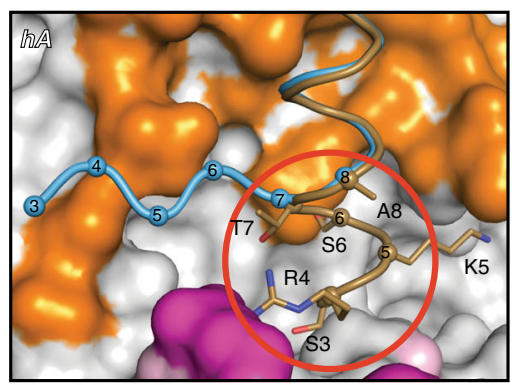

C

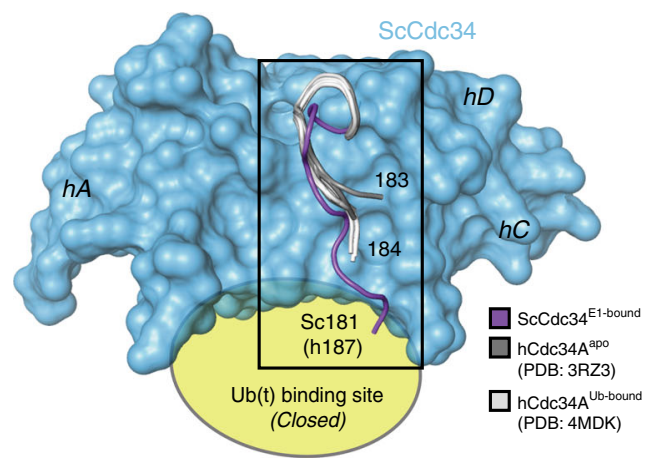

$\underline{h C d c 34 A \text { structural variants: }}$

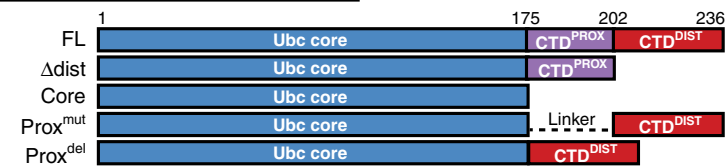

Apo vs E1-Bound ScCdc34
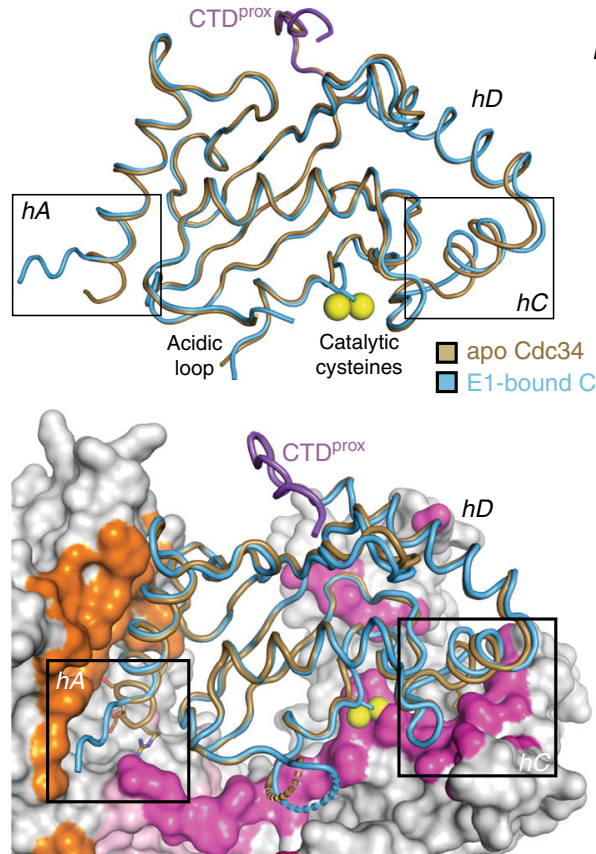

\section{Cdc34 hC/hD changes upon Uba1-binding}
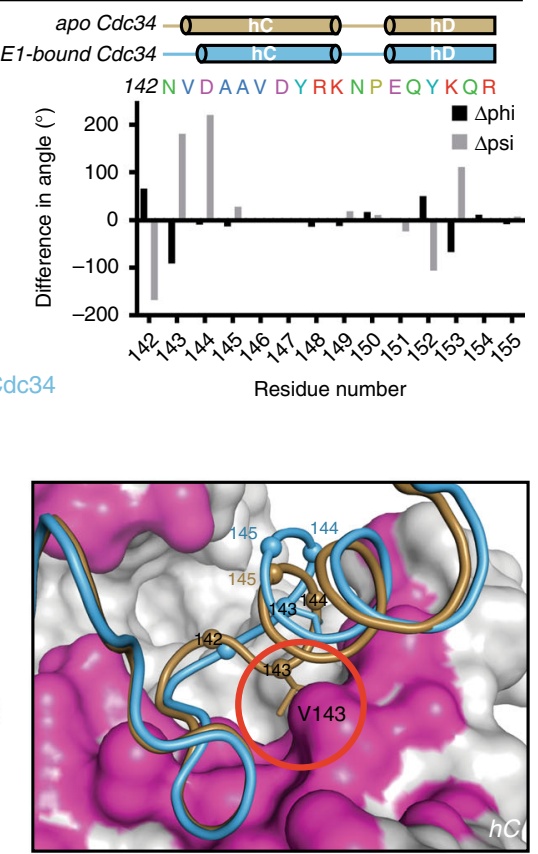

d $\quad h$ Cdc34A multiturnover Lys48 diUbiquitination assay
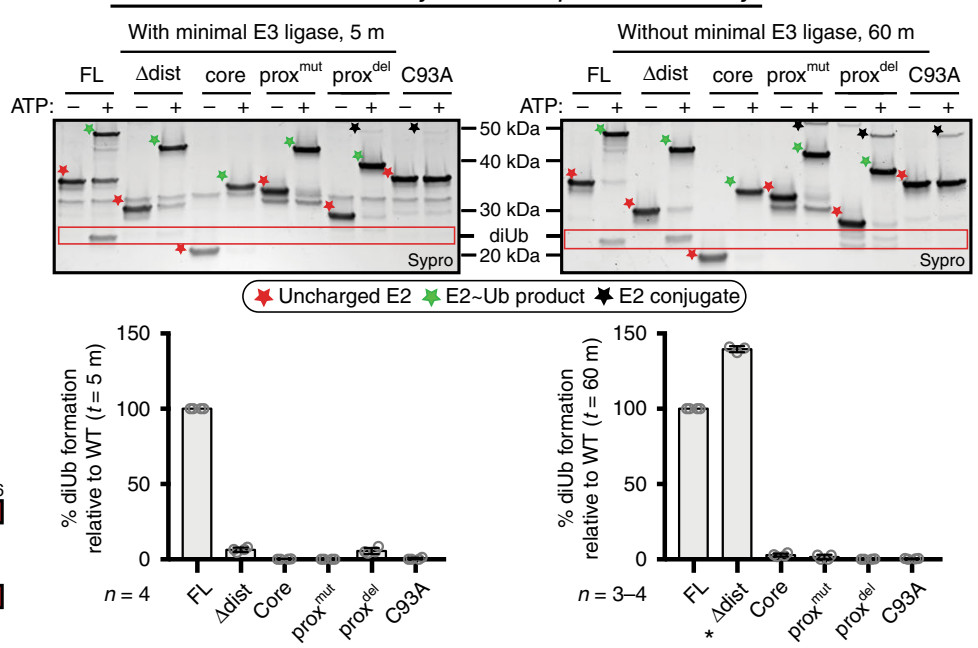

Fig. 5 Structural changes in key Cdc34 elements accompany E1 binding. a Middle, apo (tan) and E1-bound (blue) Cdc34 superimposed using the conserved core of the protein. $\mathrm{hA}$ and $\mathrm{hC}$ conformational changes are highlighted by black boxes. Differences in phi and psi angles between Cdc34E1-bound and Cdc34 ${ }^{\text {apo }}$ are shown as bar graphs for hA (left) and hC/hD (right). Cartoons above the graphs indicate secondary structure boundaries for Cdc34apo (tan) and Cdc34 $4^{\text {E1-bound }}$ (blue). b Middle, Cdc34 ${ }^{\text {apo }}$ superimposed as in a onto Uba1-Cdc34 complex. Uba1 is colored as in Fig. 1 and Cdc34 conformational changes are highlighted as in a. Zoomed in view of Cdc34 hA (left) and hC (right) with clashes between Uba1 and Cdc34apo indicated by red circles. c Top, top-down view of CTDprox of ScCdc34E1-bound (purple), hCdc34Aapo (PDB:3RZ3, dark gray), and hCdc34AUb-bound (PDB:4MDK, light gray) superimposed as worms with $\mathrm{ScCd} 34$ core shown as blue surface, and closed $\mathrm{Ub}(\mathrm{t})$-binding site indicated by a yellow oval. Bottom, cartoon schematic of hCdc34A global mutants used for K48-diUb assays. d K48-diUb assays for the indicated mutants with (left) and without (right) minimal E3 ligase. Data are represented by mean \pm SD with individual replicates shown as gray circles. Biochemical assays have four independent replicates, unless indicated by an asterisk when there are three replicates. Representative replicates are labeled above and annotated with stars for clarity. diUb product is indicated by a label and red box.

Source data are provided as a Source Data file

beyond the $\mathrm{Cdc} 34$ backside to engage $\mathrm{Ub}(\mathrm{t})$ in a $\mathrm{Cdc} 34 \sim \mathrm{Ub}$ complex. Although a Cdc34/Ub non-covalent complex structure exists ${ }^{51}$, the Cdc34 construct lacked the full CTD ${ }^{\text {rox }}$ and, thus, a Cdc34 Ub structure that includes the CTD ${ }^{\text {prox }}$ might capture this putative $\mathrm{CTD}$ prox $/ \mathrm{Ub}(\mathrm{t})$ interaction.

To first test the roles of the CTDprox and CTD dist in $\mathrm{Ub}(\mathrm{t})$ discharge, several human Cdc34A mutant constructs were cloned and subjected to a modified multiple-turnover Lys48-linked diubiquitination (K48-diUb) assay ${ }^{57}$ (Fig. 5d and Supplementary Fig. 5). Briefly, Cdc34 is continuously loaded with Ub by Uba1, with or without minimal SCF E3, in the presence of excess ubiquitin to allow formation of Lys48-linked diUb by Cdc34. The specificity of this assay for Lys48-linked diUb is demonstrated by utilizing K48R Ub in place of WT Ub (Supplementary Fig. 6). The tested variants 
include: $\mathrm{Cdc} 34^{\Delta \text { dist }}$ containing full CTD ${ }^{\text {prox }}, \mathrm{Cdc} 34^{\text {core }}$ containing only the Ubc core domain of the protein, and prox ${ }^{\text {mut }}$ and prox ${ }^{\text {del }}$ where the CTDProx region is either mutated to a flexible linker or deleted, respectively (Fig. $5 c$, bottom). Interestingly, in the absence of E3, only WT and Cdc $34^{\Delta \text { dist }}$ were able to produce $\mathrm{K} 48$-diUb and produced similar amounts (Fig. 5d, right). In the presence of E3, only WT Cdc34 was capable of producing K48-diUb under the tested assay conditions, suggesting that the CTD ${ }^{\text {rox }}$ and CTD ${ }^{\text {dist }}$ regions are both critical for productive $\mathrm{E} 3$ interaction and catalysis of $\mathrm{Cdc} 34 \mathrm{Ub}(\mathrm{t})$ discharge (Fig. 5d, left). At a longer timepoint with $\mathrm{E} 3, \mathrm{Cdc} 34^{\Delta \text { dist }}$ appears to behave as though there is no E3 in the reaction, suggesting that the deficiency observed at $5 \mathrm{~m}$ is due only to the lack of E3 catalysis (Supplementary Fig. 5). Under these assay conditions, E2 Ub formation is not affected by these mutations, indicating that a defect in E1-E2 thioester transfer does not contribute to downstream effects on K48-diUb formation (Fig. 5d and Supplementary Fig. 5). Thus, CTDprox is necessary and sufficient for the inherent ability of $\mathrm{Cdc} 34$ to discharge $\mathrm{Ub}$ while both the CTD prox and CTD ${ }^{\text {dist }}$ are necessary for productive E3 catalysis. Comparatively, equivalent mutants of yeast Cdc34 display a similar phenotype in the presence of E3 catalysis (Supplementary Fig. 5). Additionally, under E3-free conditions the yeast Cdc34 mutants also exhibit a reduction in activity, though it is less severe (Supplementary Fig. 5). Altogether, these observations support the hypothesis that the CTDProx contacts $\mathrm{Ub}(\mathrm{t})^{49}$ and is consistent with previous modeling studies that suggested the acidic CTD dist binds a basic canyon on $\mathrm{Cul1}^{46}$.

Cdc34 CTDprox locks $\mathrm{Ub}(\mathrm{t})$ in the closed conformation. To elucidate the mechanism by which CTDprox enhances Cdc34 activity, a crystal structure of a Cdc34 Ub(t) complex that includes a complete CTDprox is needed. To overcome the inherent instability of the $\mathrm{Cdc} 34 \sim \mathrm{Ub}$ thioester bond, a human $\mathrm{Cdc} 34 \mathrm{~B}^{\Delta \text { dist }_{\sim}} \mathrm{Ub}$ mimetic (Cdc34-Ub, where - indicates an isopeptide linkage) was produced wherein a stable isopeptide bond between Cdc34 active site Cys $\rightarrow$ Lys mutant and the Ub(t) Cterminal glycine replaces the labile thioester bond, using a modification of a previously described protocol ${ }^{45,58}$. A $1.8 \AA$ structure of Cdc34-Ub was determined in the presence of Cdc34 inhibitor, CC0651, which previous studies show stabilizes the interaction between $\mathrm{Ub}(\mathrm{t})$ and the closed binding surface on Cdc34 (ref. ${ }^{51}$ ) (Fig. 6a, b and Supplementary Table 1). Analysis of the overall Cdc34-Ub structure shows that, as anticipated, the complex adopts the closed conformation with $\mathrm{Ub}(\mathrm{t})$ engaging the crossover helix (hB) of Cdc34 (Fig. 6a, b). The Cdc34 acidic loop insertion is ordered in this structure, but aside from contacts to $\operatorname{Arg} 74$, it does not extensively interact with $\mathrm{Ub}(\mathrm{t})$ and is likely ordered due to crystal contacts. Additionally, the C-terminus of $\mathrm{Ub}$ is completely ordered through the isopeptide bond with Cdc34 Lys93 (Supplementary Fig. 6). Importantly, comparison of the $\mathrm{Cdc} 34-\mathrm{Ub}$ structure to the previously determined noncovalent hCdc34A/Ub structure (PDB: $4 \mathrm{MDK}$ ) reveals that eight additional residues of $\mathrm{CTD}^{\text {prox }}$ become ordered and engage in a network of contacts with $\mathrm{Ub}(\mathrm{t})$ that fortify the Cdc34/Ub closed interface (Fig. 6a, right; 6b, right). Previous studies indicate that the closed conformation of $\mathrm{E} 2 \sim \mathrm{Ub}$ is the active form of the complex and one key role of canonical RING E3 is to lock E2 Ub in the closed conformation that is primed for Ub discharge $e^{43}$. Thus, analysis of our Cdc34-Ub complex suggests that the structural basis by which CTDprox enhances Cdc34 activity is stabilization of the Cdc34 Ub closed conformation.

Detailed analysis of the Cdc34-Ub structure reveals that CTDprox engages in an extensive network of intramolecular interactions with the backside of $\mathrm{Cdc} 34$ that is known to bind non-covalent $\mathrm{Ub}$ to allosterically enhance activity in a subset of
E2s ${ }^{59}$ (Fig. 6b and Supplementary Fig. 6). The network of contacts between CTDprox and the Cdc34 core involves Val181, Val183, Pro184, and Thr185 of CTDprox and Phe46, Pro49, Glu54, Gly55, Tyr57, and Ala176 of the core. Val181 contacts both Phe46 and Tyr57 of the core and forms a backbone hydrogen bond to Ala176. Val183 contacts Phe46, Gly55, and Tyr57, while Pro184 primarily contacts Phe46 (Fig. 6b, right). Finally, Thr185 contacts Pro49 and Glu54 of the core. Single mutations of any of the central residues at this interface: CTDprox Val181, Val183, and Pro140, as well as core Phe46 and Tyr57, results in near total loss of $\mathrm{K} 48$-diUb activity in the presence of E3 and severe reduction of activity in the absence of E3 (Fig. 6c, top and middle). This highlights the importance of this intramolecular network of interactions for efficient Ub discharge by $\mathrm{Cdc} 34$, with or without E3 catalysis.

The intramolecular interactions between Cdc34 CTDprox and the backside of the Cdc34 core guide the CTDprox toward the closed Ub (t) where intermolecular interactions occur. These interactions involve a short $\alpha$-helix ( $\mathrm{hE}$ ) in the additionally ordered region of CTDprox . Tyr190 of Cdc34 CTDprox inserts into a composite pocket formed by residues from both $\mathrm{Ub}(\mathrm{t})$ and the $\mathrm{Cdc} 34$ core. Specifically, Tyr190 engages in interactions to $\mathrm{Ub}(\mathrm{t})$ Ala46 and Gly47 and Cdc34 core residues Glu26, Gly27, Phe46, and Pro49. Additionally, CTD prox Cys191 contacts Ub(t) Ala46, while CTDprox Leu187 contacts Ub(t) Phe45 and Ala46 and participates in longerrange contacts to $\mathrm{Ub}(\mathrm{t})$ Tyr59 and Asn60 (Fig. 6b, right). In K48diUb assays, mutation of Tyr190 to either Asp or Ala completely abolishes K48-diUb formation in the presence of E3 and nearly abolishes activity in the absence of E3 (Fig. 6c, top and middle). Further, mutation of Cdc34 Leu187 and Cys190 also significantly impairs K48-diUb activity in the presence and absence of E3 (Fig. 6c, top and middle). These data suggest a key mechanistic role for Cdc34 CTDprox in stabilizing the Cdc34 Ub closed conformation that is primed for $\mathrm{Ub}(\mathrm{t})$ discharge.

Previous studies of $\mathrm{Cdc} 34$ and other E2s indicate that there are two surfaces on Cdc34 that play an important role in polyUb chain formation: one surface that interacts with $\mathrm{Ub}(\mathrm{t}$ ) (the donor $\mathrm{Ub}$, $\mathrm{Ub}^{\mathrm{D}}$ ) to prime the active site for catalysis and a second that is involved in proper positioning of the acceptor $\mathrm{Ub}\left(\mathrm{Ub}^{\mathrm{A}}\right)$ lysine for attack of the Cdc34 Ub ${ }^{D}$ thioester bond ${ }^{60-64}$. Thus, to probe the importance of interactions between Cdc34 CTD prox and Ub(t) from the $\mathrm{Ub}(\mathrm{t})$ side of the complex, an adapted version of a previously described single turnover diUb formation assay ${ }^{62}$ was utilized. Unlike the multiturnover K48-diUb assay, the single turnover K48diUb assay can distinguish between $\mathrm{Ub}$ residue roles as a donor versus an acceptor. Additionally, as for the multiple-turnover assay, the single turnover assay specifically produces Lys48-linked diUb (Supplementary Fig. 6). Consistent with the Cdc34-Ub structure, F45D, A46D, and G47D Ub mutants each exhibit a significant decrease in K48-diUb formation when used as $\mathrm{Ub}^{\mathrm{D}}$ under single turnover assay conditions due to disruption of interactions with CTDprox (Fig. 6c, bottom). As might be expected based on the proximity to Lys48, F45D, A46D, and G47D mutants also exhibit a significant loss of $\mathrm{K} 48$-diUb activity when used as $\mathrm{Ub}^{\mathrm{A}}$, likely due to disruption of contacts required for proper positioning of $\mathrm{Ub}^{\mathrm{A}} \mathrm{Lys} 48$ during attack of the Cdc34 $\mathrm{Ub}^{\mathrm{D}}$ thioester bond (Fig. 6c, bottom). Altogether, these data further validate the importance of the identified interactions between the $\mathrm{Ub}(\mathrm{t})$ and $\mathrm{Cdc} 34$ CTDprox in fortifying the Cdc34 Ub closed conformation (Fig. 6b) and confirm the importance of this loop in positioning $\mathrm{Ub}^{\mathrm{A}}$ Lys48 for nucleophilic attack during K48-polyUb formation.

CTD prox/Ub contacts are important for Cdc34 action in cells. We next examined the functional importance of Cdc34 CTDprox interactions with $\mathrm{Cdc} 34$ core and $\mathrm{Ub}(\mathrm{t})$ in mammalian cells; 
a
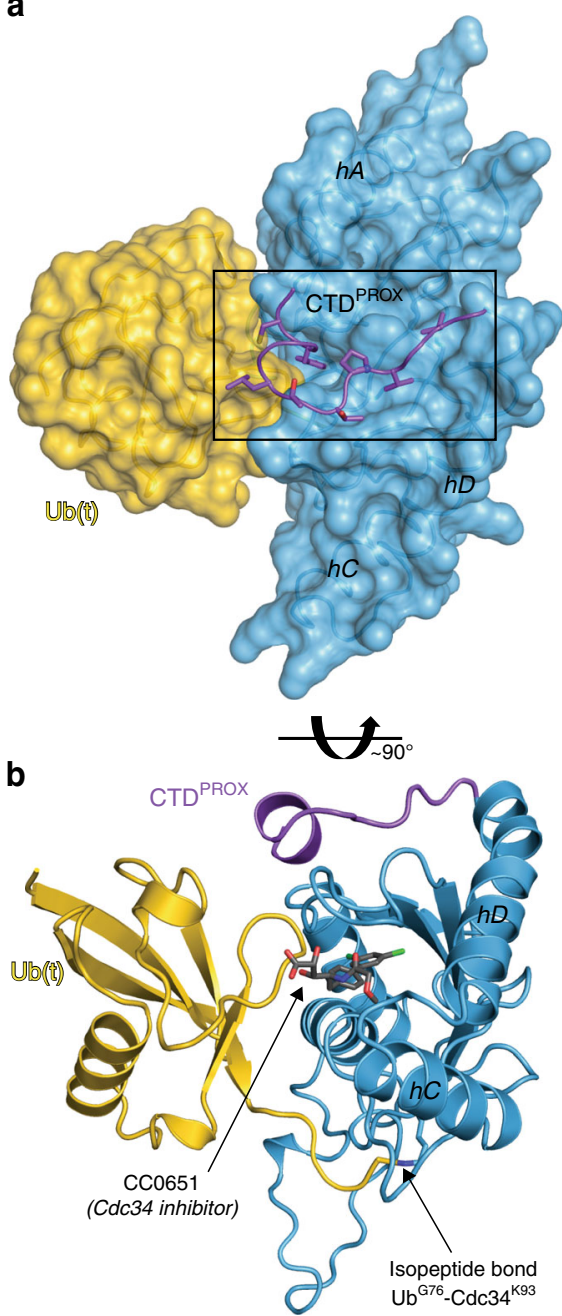
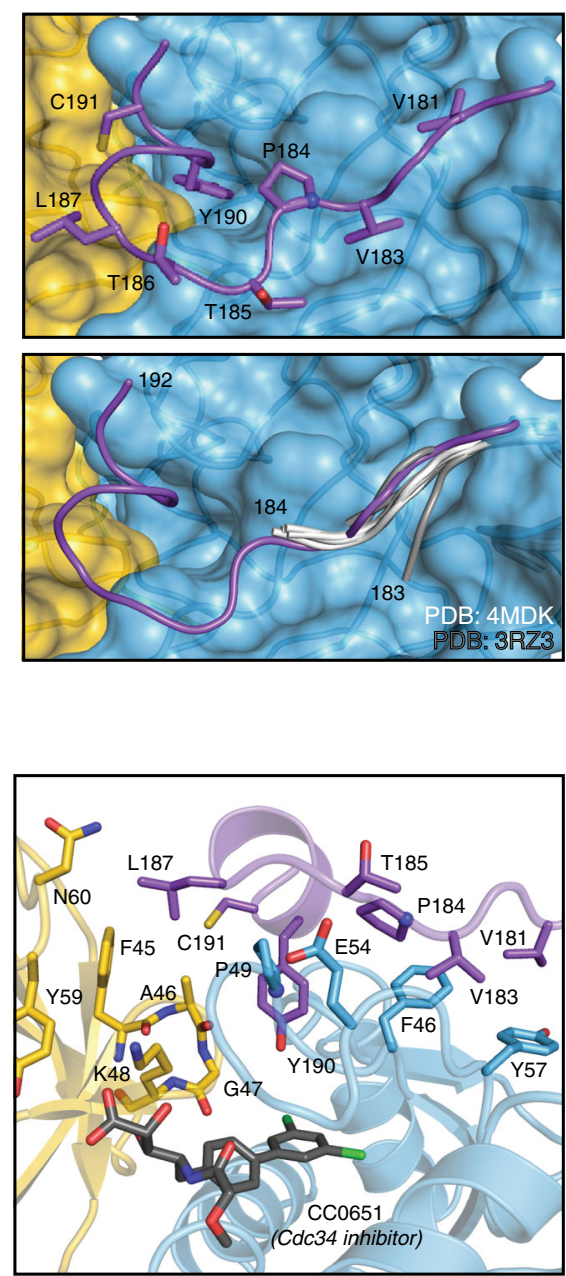

C Multiple turnover K48-diUb assay With minimal E3 ligase, $5 \mathrm{~m}$
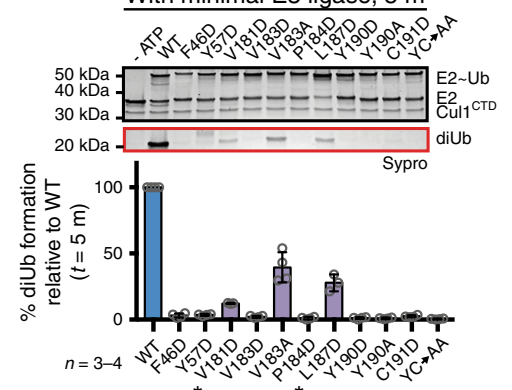

Without minimal E3 ligase, $60 \mathrm{~m}$

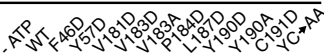
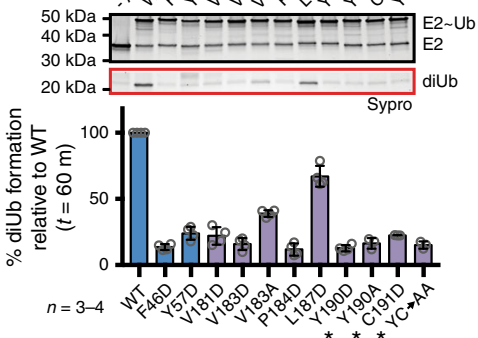

Single turnover K48-diUb assay
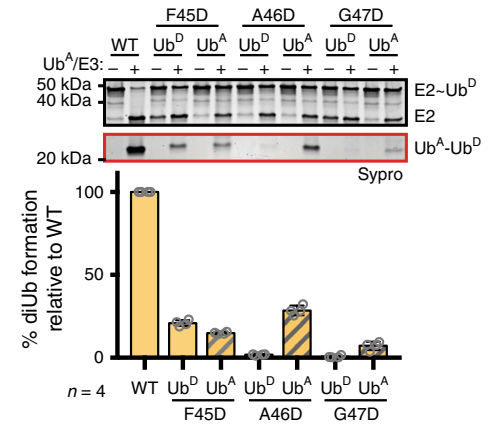

Fig. 6 An ordered Cdc34 CTDprox extension is involved in Ub discharge. a Left, crystal structure of hCdc34B ${ }^{\Delta \text { dist }}$ conjugated to Ub (gold) at active site Cys to Lys mutation in presence of CC0651 inhibitor, shown as in Fig. 5c but rotated 90 . Top right, zoomed in view of Cdc34 CTDprox trajectory over the Ubc core with contacting residues shown as sticks. Bottom right, zoomed in view of CTDprox as in Fig. 5c. $\mathbf{b}$ Left, cartoon representation of hCdc34B 4 dist_Ub as in a but rotated $\sim 90^{\circ}$ with CC0651 shown as gray sticks and isopeptide bond indicated. Right, zoomed in view of Cdc34 CTD prox, Ubc core, and Ub(t) interfaces with contacting residues shown as sticks. c K48-diUb assays for the indicated mutants with (top) and without (middle) minimal E3 ligase. Data are represented by mean \pm SD with individual replicates shown as gray circles. Biochemical assays have four independent replicates, unless indicated by an asterisk when there are three replicates. Representative replicates are labeled above. Bottom, single turnover K48-diUb assay with indicated Ub mutants ( $n$ $=4)$. Data are shown as above with representative image labeled above with $(-)$ lanes to show initial thioester formation and $(+)$ to indicate subsequent addition of E3 ligase and excess Ub ${ }^{\mathrm{A}}$ for single Ub discharge. Source data are provided as a Source Data file

specifically, control of cell cycle progression via K48-polyUbmediated degradation of cyclin-dependent kinase (CDK) inhibitors $^{65}$. To test this, we selected three Cdc34A mutants with diminished activities in vitro (Figs. 5d and 6c), including V183D, Y190D/ $\mathrm{C191D}(\mathrm{YC} \rightarrow \mathrm{DD})$, and the prox ${ }^{\text {mut }}$ construct with the CTDprox replaced by a flexible linker. As expected, knockdown (KD) of Cdc34A in U2OS human osteosarcoma cells using two independent short-hairpin RNAs (shRNAs) led to increased p27 levels ${ }^{66}$ (Fig. 7a and Supplementary Fig. 7). Furthermore, overexpression (OE) of WT Cdc34 reversed p27 protein elevation in Cdc34 KD cells, while $\mathrm{OE}$ of Cdc34 mutants V183D, YC $\rightarrow \mathrm{DD}$, and prox ${ }^{\text {mut }}$ were not able to do so (Fig. 7b and Supplementary Fig. 7). To study the potential effects of Cdc34 CTDprox disruption on cell cycle progression, U2OS cells were subjected a flow cytometry-based cell cycle assay. First, U2OS cells treated with control or Cdc34 shRNA were synchronized in G2 phase (Fig. 7c, left) followed by release for twelve hours (Fig. 7c, right). DNA content was tracked by propidium iodide (PI) incorporation combined with flow cytometric analysis, and gates were indicated for G1 (magenta), S (blue), and G2/M (cyan) phases. Upon release, more cells with $\mathrm{Cdc} 34 \mathrm{KD}$ were retained in $\mathrm{G} 1$ phase with an increase from 37 to $45 \%$ compared to control cells (Fig. 7c, d). Although these differences were subtle, they were consistent over three independent experiments and were statistically significant (Fig. 7d), suggesting that the observed retention of live cells in G1 is indeed due to Cdc34A KD in the cells that may be partially compensated for by the Cdc34B isoform or even other E2s. Subsequently, U2OS cells with Cdc34 KD plus OE of WT or mutant $\mathrm{Cdc} 34$ were subjected to the same flow cytometric analysis. OE of WT Cdc34 was able to rescue the increase of cells in G1 phase from $\mathrm{Cdc} 34 \mathrm{KD}$; whereas, OE of Cdc34 mutants was unable to rescue this effect, consistent with the earlier in vitro findings (Fig. $7 \mathrm{~d}$, e). Interestingly, U2OS cells expressing the Cdc34 mutants V183D, $\mathrm{YC} \rightarrow \mathrm{DD}$, and prox $^{\text {mut }}$ displayed a phenotype similar to cells expressing catalytically inactive Cdc34 mutant, C93A (Fig. 7d, e). 
a

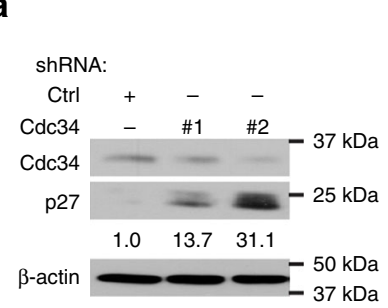

b

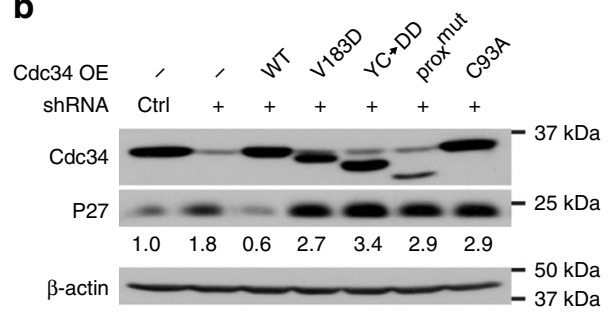

e
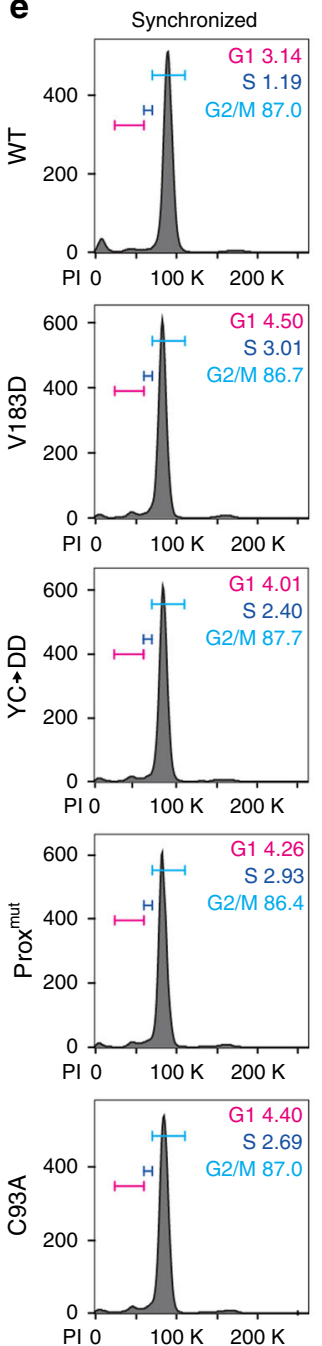
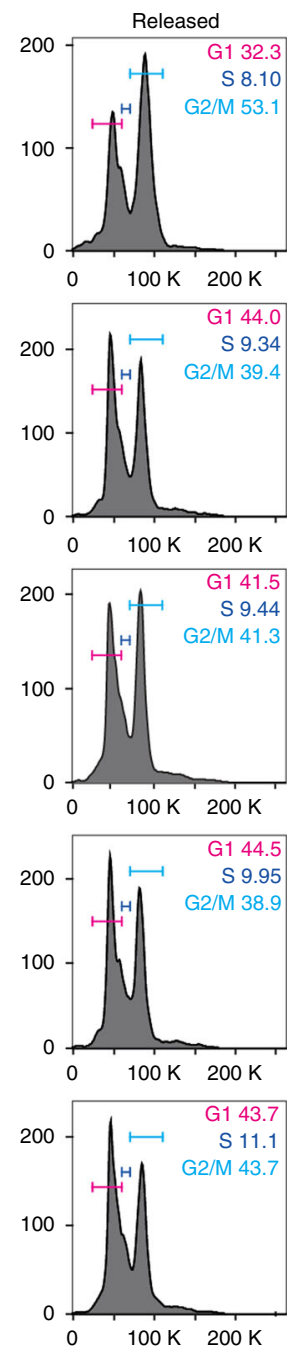

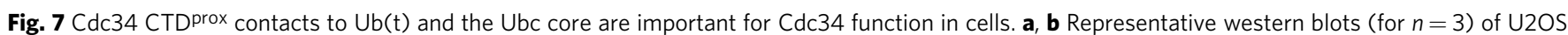
cells for Cdc34 and p27 after Cdc34 shRNA KD (a) and subsequent overexpression of WT or mutant Cdc34 (b). c Flow cytometric analysis of U2OS cells treated with control or Cdc34 shRNA then synchronized in G2 phase with nocodazole (middle) and released from synchrony for $12 \mathrm{~h}$ (right) with propidium iodide staining to track DNA content. Left, control asynchronous U2OS cell population subjected to the same analysis. Gates indicate percent of live cell population in stages of cell cycle indicated. $\mathbf{d}$ Graphical representation of three independent cell cycle experiments as in c and e with the percent of live cells in G1 phase during G2 synchronization (left) and after 12-h release (right) represented by the mean \pm SD with individual replicates shown as gray circles. Statistical analysis was conducted using a one-way ANOVA with Bonferroni as Post Hoc test ( $n=3$ ), and n.s. indicates non-significant changes while $^{\star \star \star}$ indicates a $p$-value of $<0.001$. e Flow cytometric analysis as in c with Cdc34 shRNA KD followed by WT or mutant Cdc34 overexpression as indicated by labels. Source data for all panels are provided as a Source Data file

These findings suggest that the selected Cdc34 mutants are defective in polyubiquitination, leading to the accumulation of p27 and slowed progression of cell cycle. While the structural and biochemical data strongly suggest that this phenotype is due to the disruption of inherent Cdc34 CTDProx function by destabilizing the $\mathrm{Ub}(\mathrm{t})$ closed conformation, other potential mechanisms may exist in the cell such as the disruption of Cdc34/SCF binding and further study is required to dissect the detailed molecular mechanisms. Altogether, the results of the structural, biochemical, and cell-based assays support the importance of intra- and intermolecular CTDprox interactions for Cdc34 K48-polyUb chain formation on protein targets in cells and, subsequently, regulation of cell cycle progression.

\section{Discussion}

In this manuscript, we have presented a series of structural snapshots that mimic the substrate complex and product of
Uba1-Cdc34 Ub thioester transfer. Our Cdc34apo and Uba1-Cdc34 structures reveal combinatorial Ubal recognition of Cdc34 involving a distinct Cdc34/UFD interface and conformational changes in Cdc34 that accompany Ubal binding. Comparative analysis reveals that the Ubal UFD utilizes a conserved core of residues to build distinct networks of contacts with divergent E2 hA sequences, highlighting UFD plasticity. Further, a flexible Uba1 architecture allows E1 and E2 active sites to come together during thioester transfer despite significant differences $\mathrm{i} \sim \mathrm{n}$ E2-binding modes. These findings could provide broader insights into the molecular basis for promiscuity/specificity in protein-protein interactions, particularly with regard to protein adaptation to dynamic environments and how evolution shapes protein interaction networks.

Our Cdc34-Ub structure reveals that elements of the Cdc34 CTD prox become ordered upon transfer of $\mathrm{Ub}$ to $\mathrm{Cdc} 34$ and engage in contacts to $\mathrm{Ub}(\mathrm{t})$ that stabilize an active $\mathrm{Cdc} 34 \sim \mathrm{Ub}$ 
closed conformation. This discovery answers a longstanding question regarding the structural basis by which CTD prox promotes Cdc34 activity. Notably, Cdc34/Ub(t) interactions are facilitated by intramolecular interactions between CTDProx and the previously identified E2 backside surface that interacts with non-covalent Ub for allosteric activation in a subset of E2s. Many E2s harbor uncharacterized C-terminal extensions and our studies raise the possibility that these regions may also play a role in E2 activity through contacts to $\mathrm{Ub}(\mathrm{t})$ or allosteric activation. Altogether our structural, biochemical, and cell-based studies reveal the molecular underpinnings for two critical Cdc34 activities: engaging Ubal for E1-E2 thioester transfer and positioning of the subsequent thioester-bound $\mathrm{Ub}$ molecule for optimal Ub discharge for Lys48-linked polyUb chain extension. These findings provide a step forward in the progress of understanding Cdc34's cellular functions and lay groundwork for future investigations of Cdc34 and SCF ligase interactions.

\section{Methods}

Cloning. S. cerevisiae Uba1(a.a. 11-1024) and $\mathrm{Cdc} 34^{\mathrm{FL}}$ were cloned from genomic DNA into the NcoI and XhoI sites of pET29NTEV. Human Cdc34A $\mathrm{A}^{\mathrm{FL}}$ and $\mathrm{Cdc} 34 \mathrm{~B}^{\mathrm{FL}}$ were amplified from a cDNA library and cloned into the NcoI and XhoI sites of pET29NTEV. Human and $S$. pombe Ubal were cloned as previously described $^{33}$. S. pombe ubiquitin was cloned into pET28 with a non-cleavable Nterminal His tag. Human ubiquitin was cloned from cDNA into the NcoI and XhoI sites of pET29NTEV. All point mutations were introduced using PCR-based sitedirected mutagenesis. Truncation mutants of $\mathrm{Cdc} 34$ were introduced by mutating a stop codon at the desired site. Mutated and deleted CTD prox constructs were synthesized then inserted into the NcoI and XhoI of pET29NTEV. Human Cull (a. a. 411-690) and S. cerevisiae Cull (a.a. 429-814) were synthesized and inserted in the BamHI and NotI sites of pSMT3. Human Rbx1 (a.a. 5-108) and S. cerevisiae Rbxl (1-121) were cloned from a synthesized construct and inserted into NcoI and XhoI of pETduet. All constructs and point mutations were generated using the primer pairs described in Supplementary Table 2.

Protein expression and purification. All proteins were expressed in $E$. coli strain BL21 (DE3) Codon Plus (Stratagene). Rbx1 and Cull constructs were co-expressed using dual antibiotic selection. Large-scale cultures were grown at $37^{\circ} \mathrm{C}$ until the desired A600 OD was reached then placed in an ice bath cold shock with $1.5 \%$ ethanol. After $30 \mathrm{~min}$, isopropyl- $\beta$-D-1-thiogalactoside (IPTG) was added to a desired concentration of $0.1-0.5 \mathrm{mM}$, then protein expression was induced at $18^{\circ} \mathrm{C}$ shaking for $18 \mathrm{~h}$. At induction, $0.15 \mathrm{mM}$ zinc acetate was added to Cull/Rbx1 coexpression cultures. All proteins were grown in Luria Broth to an OD of 1.0-2.0 except for S. cerevisiae Uba1, which was grown to saturation in Terrific Broth overnight prior to induction. After induction, cells were pelleted by centrifugation then resuspended in $20 \mathrm{mM}$ Tris $\mathrm{pH} 8.0,350 \mathrm{mM} \mathrm{NaCl}$ and $20 \mathrm{mM}$ imidazole and snap frozen in liquid nitrogen for storage or processed immediately. Frozen pellets were rapidly thawed then $0.5 \mathrm{mM}$ dithiothreitol (DTT), $100 \mu \mathrm{g}$ DNase per liter of culture, and $1 \mathrm{mg}$ Lysozyme per liter of culture were added and cells were lysed by sonication. Cell lysis supernatant was cleared by centrifugation then applied to $\mathrm{Ni}$-NTA superflow resin (QIAGEN) by gravity flow. Bound protein was eluted in $20 \mathrm{mM}$ Tris $\mathrm{pH}$ 8.0, $350 \mathrm{mM} \mathrm{NaCl}, 250 \mathrm{mM}$ imidazole, $2 \mathrm{mM}$ DTT. Purified protein was subjected to gel filtration by Superdex 200 or Superdex 75 based on protein size (Amersham), tag cleavage overnight where applicable, anion exchange purification of a MonoQ column (Amersham), then buffer exchange to $20 \mathrm{mM}$ Tris-HCl pH 8.0, $50 \mathrm{mM} \mathrm{NaCl}$. The exception to this protocol were Cdc34 WT and mutants and SpUb mutants used for biochemistry. These proteins were purified using limiting amounts of Ni-NTA resin then passed over a desalting column (Amersham) for buffer exchange to $20 \mathrm{mM}$ Tris- $\mathrm{HCl} \mathrm{pH} 8.0,50 \mathrm{mM} \mathrm{NaCl}$. After purification, proteins were concentrated to $4-10 \mathrm{mg} \mathrm{ml}^{-1}$, aliquoted for single use, and snap frozen in liquid nitrogen.

E1-E2 cross-linking. E1-E2 cross-linking was performed according to published methods ${ }^{32,33}$. Cdc34 was incubated in fresh activating buffer $(20 \mathrm{mM}$ Tris $\mathrm{pH}$ 8.0,

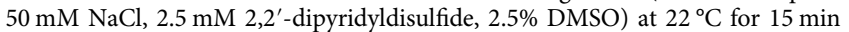
followed by filtration and desalting to removed excess $2,2^{\prime}$-dipyridyldisulfide. Then, Ubal and activated Cdc34 were mixed and incubated for $15 \mathrm{~min}$ at $22^{\circ} \mathrm{C}$. UbalCdc34 crosslink product was purified over non-reducing Superdex 200 and MonoQ columns, concentrated to $11.3 \mathrm{mg} \mathrm{ml}^{-1}$ and snap frozen in liquid nitrogen.

Cdc34-Ub conjugation. Five micromolar Cdc34 active site Cys to Lys mutant was incubated with $0.5 \mu \mathrm{M}$ Ubal and $50 \mu \mathrm{M}$ Ub overnight at $35^{\circ} \mathrm{C}$ in $50 \mathrm{mM}$ Tris- $\mathrm{HCl}$ $\mathrm{pH} 9.5,50 \mathrm{mM} \mathrm{NaCl}, 5 \mathrm{mM}$ ATP, $10 \mathrm{mM} \mathrm{MgCl}_{2}$, and $1 \mathrm{mM} \mathrm{BME}$. hCdc $34 \mathrm{~B}^{\Delta \text { dist }}$ Ub conjugate was purified over a MonoQ column and concentrated to $17 \mathrm{mg} \mathrm{ml}^{-1}$ then snap frozen in liquid nitrogen.
Crystallization and data collection. Prior to setting crystallization screens, $S$. cerevisiae Uba1-Cdc $34^{\Delta \text { dist }}$ crosslink $\left(8.7 \mathrm{mg} \mathrm{ml}^{-1}, 58 \mu \mathrm{M}\right.$, final) was mixed with $116 \mu \mathrm{M} \mathrm{Ub}, 1 \mathrm{mM}$ ATP, and $5 \mathrm{mM} \mathrm{MgCl}_{2}$. Sample was subjected to sparse-matrix screening in Intelli-Plate (Art Robbins Instruments) sitting drop format with $0.2 \mu$ sample and $0.2 \mu \mathrm{l}$ mother liquor at $18^{\circ} \mathrm{C}$. Crystals initially grew in the condition F7 from Index (Hampton Research). Refinements of crystal conditions in a hanging drop vapor diffusion tray with $0.75 \mu \mathrm{l}$ of sample and $0.75 \mu \mathrm{l}$ of mother liquor resulted in large crystals growing in $0.2 \mathrm{M}$ ammonium sulfate, 25\% PEG 3,350, 0.1 $\mathrm{M}$ Bis-Tris $\mathrm{pH}$ 6.5. Crystals were equilibrated in mother liquor with cryoprotectant $20 \%$ ethylene glycol, $1 \mathrm{mM} \mathrm{ATP}$, and $5 \mathrm{mM} \mathrm{MgCl}_{2}$ and snap frozen in liquid nitrogen for shipment. Data were collected at Advanced Photon Source (APS, Argonne, Illinois, USA), NE-CAT beamline 24-ID-E.

S. cerevisiae $\mathrm{Cdc} 34^{\Delta \text { dist }}\left(10 \mathrm{mg} \mathrm{ml}^{-1}\right)$ was subjected to sitting drop screens as above. Initial crystal hit was in F7 from JCSG Core Suite IV (Hampton Research). Condition refinement in a hanging drop diffusion system produced crystals in 0.06 $\mathrm{M}$ zinc acetate, $0.108 \mathrm{M}$ sodium cacodylate, $14.4 \%$ PEG 8,000, 20\% glycerol. Crystals were snap frozen for shipment. Data were collected at APS SER-CAT beamline 22-ID.

Prior to setting crystallization screens, hCdc34B $\mathrm{B}^{\Delta \text { dist }}$-Ub conjugate $(737 \mu \mathrm{M})$ was mixed with 1.33-fold excess CC0651 $(981 \mu \mathrm{M})$, Cdc34 inhibitor, as a crystallization aid. Sitting drops screens were set as above. Crystals initially grew in H4 from JCSG Core Suite I (Hampton Research). Refinement in hanging drop system produced crystals in $0.03 \mathrm{M}$ potassium dihydrogen phosphate, $23 \%$ PEG 8000. Crystals were equilibrated in mother liquor with cryoprotectant $20 \%$ ethylene glycol and snap frozen for shipment. Data were collected at APS NE-CAT beamline 24-ID-E.

Structure determination and refinement. A complete dataset for $S$. cerevisiae Uba1-Cdc34 ${ }^{\Delta \text { dist }}$ was collected to $2.07 \AA$ resolution. PHASER was used for molecular replacement to place Uba1/Ub(a) using PDB: $3 \mathrm{CMM}$ as the search model. Clear density was visible for E2, so SCULPTOR was used to create a model of ScCdc $34^{\Delta \text { dist }}$ using Ube2g2 (PDB: $3 \mathrm{H} 8 \mathrm{~K}$ ), which was placed into the available density. After refinement, clear positive density was visible for the extended Cterminus of $\mathrm{Cdc} 34$ so residues 171-181 were manually built into the density. The model was refined to $R / R_{\text {free }}$ values of $0.187 / 0.217$ via iterative rounds of refinement and rebuilding using PHENIX ${ }^{67}$ and COOT $^{68}$ and geometry analysis via MolProbity ${ }^{69}$. The final model contains two copies of Uba1-Cdc34/Ub(a) in the asymmetric unit with residues 11-773 and 797-1024 of Ubal (chains A/D), 1-76 of $\mathrm{Ub}$ (chains B/E), and 3-102 and 112-181 of Cdc34 ${ }^{\Delta \text { dist }}$ (chains C/F) built. The model also includes 909 ordered water molecules, 21 ethylene glycol molecules, 15 sulfate ions, 2 magnesium ions, and 2 ATP molecules. The space group is $\mathrm{P} 21$ with dimensions $(\AA) a=126.4, b=68.5$, and $c=171.7$.

A complete dataset for $S$. cerevisiae Cdc $34^{\Delta \text { dist }}$ was collected to $1.65 \AA$ resolution. Chain $\mathrm{C}$ from the Uba1-Cdc34/Ub(a) model was used in PHASER for molecular replacement. The model was refined to $R / R_{\text {free }}$ values of $0.171 / 0.199$ via iterative rounds of refinement and rebuilding using PHENIX and COOT. The final model contains one copy of Cdc $34^{\Delta \text { dist }}$ asymmetric unit with residues 3-102 and 113-178 ordered. The model includes 103 ordered water molecules, $4 \mathrm{Zn}^{2+}$ molecules, and 3 acetate molecules. The space group is $\mathrm{P} 2{ }_{1} 2_{1} 2_{1}$ with dimensions (A) $a=40.1, b=49.0$, and $c=103.7$.

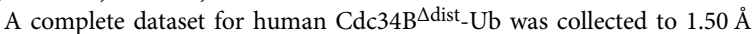
resolution. hCdc34A/Ub (PDB: $4 \mathrm{MDK}$ ) was used in SCULPTOR to create a model for molecular replacement via PHASER. After refinement, clear positive density was observed for the C-termini of $\mathrm{Ub}$ and Cdc34 so they were built into the density manually to include an isopeptide bond between Ub Gly76 and Cdc34 Lys93. The model was refined to $R / R_{\text {free }}$ values of $0.175 / 0.202$ via iterative rounds of refinement and rebuilding using PHENIX and COOT. The final model contains one copy of

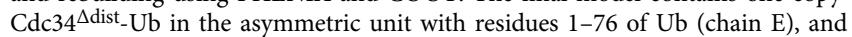
5-192 of Cdc34 ${ }^{\Delta \text { dist }}$ (chain A) built. The model also includes 319 ordered water molecules, 1 phosphate, and 3 ethylene glycol molecules. The space group is $\mathrm{P} 2{ }_{1} 2_{1} 2_{1}$ with dimensions $(\AA) a=44.6, b=55.7$, and $c=119.8$. All molecular graphics representations were generated using PYMOL. All electron density maps were constructed in PYMOL from 2Fo-Fc maps contoured at sigma level 1.0.

E1-E2 thioester transfer assay. E1-E2 thioester transfer assay was adapted from the previously described protocol ${ }^{33}$. Assays were performed with $20 \mathrm{nM}$ E1 (species-matched to E2), $500 \mathrm{nM} \mathrm{E2}, 5 \mu \mathrm{M} \mathrm{Ub}$, and $1 \mathrm{mM}$ ATP in $20 \mathrm{mM}$ HEPES pH $7.5,50 \mathrm{mM} \mathrm{NaCl}, 5 \mathrm{mM} \mathrm{MgCl} 2$ buffer. Reaction was started by addition of ATP and mixing then quenched after $30 \mathrm{~s}$ by addition of $2 \mathrm{x}$ UREA sodium dodecyl sulfate polyacrylamide gel electrophoresis (SDS-PAGE) buffer. Owing to inherently low activity, Ubc15 thioester transfer assay was conducted with $50 \mathrm{nM}$ E1 for $60 \mathrm{~s}$. Samples $(7.5 \mu \mathrm{l}$ each) were subjected to SDS-PAGE at $150 \mathrm{~V}$. Gels were then stained with SYPRO Ruby (BioRad) and visualized with a ChemiDoc MP (BioRad). Data quantification was conducted using densitometry in ImageJ software with original unedited images. Densitometry measurements were normalized as a percentage of the control WT assay on the same gel. Data are represented as an average of 3-4 technical replicates with $+/-1$ standard deviation error bars. Unprocessed images of representative gels for all biochemical assays are provided in the Source Data file. 
Multiple-turnover Lys48-linked diUbiquitination assay. Multiple-turnover Lys48-linked diUbiquitination assay was adapted from a previously described protocol $^{57}$. Assays were performed with $50 \mathrm{nM} \mathrm{E1}$ (species-matched to E2), 500 nM E2, $5 \mu \mathrm{M} \mathrm{Ub}$ (species-matched), $+/-100 \mathrm{nM}$ minimal E3 fragment (speciesmatched) and $1 \mathrm{mM}$ ATP in $20 \mathrm{mM}$ HEPES $\mathrm{pH} 7.5,50 \mathrm{mM} \mathrm{NaCl}, 5 \mathrm{mM} \mathrm{MgCl} 2$ buffer. Reaction was started by addition of ATP and mixing then quenched after 5 , 30 , or $60 \mathrm{~m}$ as indicated by addition of $2 \mathrm{x}$ UREA SDS-PAGE buffer. Samples $(7.5 \mu \mathrm{l}$ each) were subjected to SDS-PAGE, stained, and imaged as for the thioester transfer assays. Data were quantified and represented as for the E1-E2 thioester transfer assay.

Single turnover Lys48-linked diUbiquitination assay. Single turnover Lys48linked diUbiquitination assay was adapted from the previously described protocol $^{62}$. Assays were performed with $500 \mathrm{nM} \mathrm{E1}, 5 \mu \mathrm{M} \mathrm{E2}, 5 \mu \mathrm{M} \mathrm{Ub}^{\mathrm{D}}$, and $5 \mathrm{mM}$ ATP in $20 \mathrm{mM}$ HEPES pH 7.5, $50 \mathrm{mM} \mathrm{NaCl}, 5 \mathrm{mM} \mathrm{MgCl} 2$ buffer. In this assay, human proteins were used except for S. cerevisiae WT Ub and mutants. Reaction was started by addition of ATP and mixing. After $10 \mathrm{~m}$, thioestercharged samples were taken and added to $1 / 20 x$ diluted Urea SDS-PAGE buffer, and 1 unit apyrase (Sigma) was added. After $5 \mathrm{~m}, 1 \mu \mathrm{M}$ minimal E3 fragment and $50 \mu \mathrm{M} \mathrm{Ub}{ }^{A}$ was added to the reaction with mixing and then quenched after $5 \mathrm{~m}$ by addition of $1 / 20 \mathrm{x}$ diluted Urea SDS-PAGE buffer. Samples $(0.75 \mu \mathrm{l}$ each) were subjected to SDS-PAGE, stained, and imaged as for the thioester transfer assays. Data were quantified and represented as for the E1-E2 thioester transfer.

Cell culture. U2OS (Cat. \# HTB-96) and HEK293T (Cat. \# CRL-3216) cells were purchased from the American Type Culture Collection (ATCC). Both cell lines have the authentication information from ATCC, and were tested negative for mycoplasma contamination. HEK293T cells were cultured in Dulbecco's Modified Eagle Medium containing 10\% Fetal Bovine Serum (FBS) and 1\% penicillin-streptomycin. U2OS cells were maintained in McCoy's 5 A medium with $10 \%$ FBS and $1 \%$ penicillin-streptomycin. All cells were maintained in a humidified incubator with $5 \% \mathrm{CO}_{2}$.

Transfection, virus production, and infection. For lentivirus production, lentiviral vectors were co-transfected with pMDLg/pRRE, CMV-VSVG, and RSV-Rev vectors. The pLKO.1 shRNA constructs were purchased from Dharmacon (GE Healthcare Life Sciences): TRC $c d c 34$ shRNAs.

For retrovirus production, pMX-puro empty vector, and vectors with Cdc34 WT, C93A, V183D, Y190D/C191D, and prox ${ }^{\text {mut }}$ were co-transfected with either $\mathrm{Q} \Psi$ vector. Virus supernatants were collected 48 and $72 \mathrm{~h}$ post transfection. Infections were performed using polybrene. Thereafter, cells were either used for experiments or selected with puromycin for further analyses.

Western blot analysis. After treatment, cells were collected and lysed in RIPA buffer (50 mM Tris pH 7.5, $150 \mathrm{mM} \mathrm{NaCl}, 1.0 \% \mathrm{NP}-40,0.1 \%$ SDS, and $0.5 \%$ deoxycholic acid) supplemented with protease and phosphatase inhibitors. Lysates were resolved in $10 \%$ SDS-PAGE gels for western blotting. Proteins were transferred to PVDF membrane; after blocking with $5 \%$ non-fat milk, membranes were incubated with primary antibodies: Cdc34 (Santa Cruz, sc-28381, 1:1000 dilution), p27 (BD Biosciences, 610242, 1:1000 dilution), p27 (Cell Signaling, 3686, 1:1000 dilution), SKP2 (Santa Cruz, sc-7164, 1:1000 dilution), SKP1 (BD Biosciences, 610530, 1:1000 dilution), and $\beta$-actin (Sigma, A5316, 1:50,000 dilution). Thereafter, membranes were washed and incubated with relative secondary antibodies: goat anti-rabbit secondary antibody (Cell Signaling, 7074, 1:5000 dilution), or goat anti-mouse secondary antibody (Cell Signaling, 7076, 1:5000 dilution).

Finally, the signals were visualized by the chemiluminescence system (Perkin Elmer). These experiments were conducted three times. Western blot band quantification was performed using Quantity One (BioRad Laboratories, Inc.) and signals were normalized to the control group. Unprocessed scans of blots are provided in the Source Data file.

Cell cycle analysis. U2OS cells with control shRNA or Cdc34 shRNA as well as overexpression of Cdc34 WT or mutants were exposed to nocodazole synchronization for $16 \mathrm{~h}$ and then released for $12 \mathrm{~h}$; meanwhile, asynchronized cells were used as a control population. Thereafter, cells were trypsinized and washed with cold PBS. Then, cells were stained with $10 \mu \mathrm{g} \mathrm{ml}^{-1}$ propidium iodide (PI) containing $100 \mu \mathrm{g} \mathrm{ml}^{-1}$ RNaseA before flow cytometry analysis. First, the total number of nuclei events acquired were gated using FSC-A vs. SSC-A plot; then, the singlet nuclei were gated by applying a Propidium Iodide- $\mathrm{W}$ vs. Propidium Iodide-A plot; and finally, a histogram of Propidium Iodide-A from the singlet was gated to display the phases of the cell cycle.

Statistical analysis. Measurements were taken from distinct samples, and the sample size $(n)$ is indicated in the relative figure legends. Plots were made using GraphPad Prism7. SPSS version 24.0 was used for statistical analyses. One-way
ANOVA was applied to compare means. The significance level was set at 0.05 for all analyses.

Reporting summary. Further information on research design is available in the Nature Research Reporting Summary linked to this article.

\section{Data availability}

Atomic coordinates and structure factors are deposited in the RCSB with accession codes 6 NYA, $6 \mathrm{NYD}$, and $6 \mathrm{NYO}$. The source data underlying Figs. 1a, 2b, c, e, 4b, 5d, 6c, 7a-d, Supplementary Figs. 1b, 5c, 6e, 7a, and $7 \mathrm{~b}$ are provided as a Source Data file. All other data supporting the findings of this study are available from the corresponding author upon reasonable request.

Received: 5 March 2019 Accepted: 20 June 2019

Published online: 24 July 2019

\section{References}

1. Dutcher, S. K. \& Hartwell, L. H. The role of S. Cerevisiae cell division cycle genes in nuclear fusion. Genetics 100, 175-184 (1982).

2. Goebl, M. G. et al. The yeast cell cycle gene CDC34 encodes a ubiquitinconjugating enzyme. Science 241, 1331-1335 (1988).

3. Haas, A. L., Warms, J. V., Hershko, A. \& Rose, I. A. Ubiquitin-activating enzyme. Mech. role protein-ubiquitin Conjug. J. Biol. Chem. 257, 2543-2548 (1982).

4. Haas, A. L., Reback, P. B. \& Chau, V. Ubiquitin conjugation by the yeast RAD6 and CDC34 gene products. Comparison to their putative rabbit homologs, E2(20K) AND E2(32K). J. Biol. Chem. 266, 5104-5112 (1991).

5. Mathias, N. et al. Cdc53p acts in concert with Cdc4p and Cdc34p to control the G1-to-S-phase transition and identifies a conserved family of proteins. Mol. Cell. Biol. 16, 6634-6643 (1996).

6. Pagano, M. et al. Role of the ubiquitin-proteasome pathway in regulating abundance of the cyclin-dependent kinase inhibitor p27. Science 269, 682-685 (1995)

7. Tam, S. W., Theodoras, A. M. \& Pagano, M. Kip1 degradation via the ubiquitin-proteasome pathway. Leukemia 11, 363-366 (1997).

8. Verma, R., Feldman, R. M. \& Deshaies, R. J. SIC1 is ubiquitinated in vitro by a pathway that requires CDC4, CDC34, and cyclin/CDK activities. Mol. Biol. Cell 8, 1427-1437 (1997).

9. Henchoz, S. et al. Phosphorylation- and ubiquitin-dependent degradation of the cyclin-dependent kinase inhibitor Farlp in budding yeast. Genes Dev. 11, 3046-3060 (1997)

10. Tanaka, K. et al. Enhanced expression of mRNAs of antisecretory factor-1, gp96, DAD1 and CDC34 in human hepatocellular carcinomas. Biochim. et. Biophys. Acta (BBA) - Mol. Basis Dis. 1536, 1-12 (2001).

11. Takagi, K. et al. Cell division cycle 34 is highly expressed in hepatitis $C$ viruspositive hepatocellular carcinoma with favorable phenotypes. Biomed. Rep. 7, $41-46$ (2017)

12. Eliseeva, E. et al. Expression and localization of the CDC34 ubiquitinconjugating enzyme in pediatric acute lymphoblastic leukemia. Cell Growth Differ. 12, 427-433 (2001).

13. Zeng, Y. et al. $\delta$-Catenin promotes prostate cancer cell growth and progression by altering cell cycle and survival gene profiles. Mol. Cancer 8, 19 (2009).

14. Price, G. R. et al. Phenotype-directed analysis of genotype in early-onset, familial breast cancers. Pathology 38, 520-527 (2006).

15. Macdonald, M. et al. Control of cell cycle-dependent degradation of c-Ski proto-oncoprotein by Cdc34. Oncogene 23, 5643-5653 (2004).

16. Charrasse, S., Carena, I., Brondani, V., Klempnauer, K. H. \& Ferrari, S. Degradation of B-Myb by ubiquitin-mediated proteolysis: involvement of the Cdc34-SCFP45Skp2 pathway. Oncogene 19, 2986-2995 (2000).

17. Chauhan, D. et al. Blockade of ubiquitin-conjugating enzyme CDC34 enhances anti-myeloma activity of Bortezomib/Proteasome inhibitor PS-341. Oncogene 23, 3597-3602 (2004).

18. Wei, Y. et al. Cdc34-mediated degradation of ATF5 is blocked by cisplatin. J. Biol. Chem. 283, 18773-18781 (2008).

19. Ceccarelli, D. F. et al. An allosteric inhibitor of the human Cdc34 ubiquitinconjugating enzyme. Cell 145, 1075-1087 (2011).

20. Wu, K. et al. Suramin inhibits cullin-RING E3 ubiquitin ligases. Proc. Natl Acad. Sci. 113, E2011-E2018 (2016).

21. Liu, Y. \& Mallampalli, R. K. Small molecule therapeutics targeting F-box proteins in cancer. Semin. Cancer Biol. 36, 105-119 (2016).

22. Hershko, A., Eytan, E., Ciechanover, A. \& Haas, A. L. Immunochemical analysis of the turnover of ubiquitin-protein conjugates in intact cells. Relationship to the breakdown of abnormal proteins. J. Biol. Chem. 257, 13964-13970 (1982) 
23. Lv, Z. et al. Domain alternation and active site remodeling are conserved structural features of ubiquitin E1. J. Biol. Chem. 292, 12089-12099 (2017).

24. Lake, M. W., Wuebbens, M. M., Rajagopalan, K. V. \& Schindelin, H. Mechanism of ubiquitin activation revealed by the structure of a bacterial MoeB-MoaD complex. Nature 414, 325-329 (2001).

25. Lois, L. M. \& Lima, C. D. Structures of the SUMO E1 provide mechanistic insights into SUMO activation and E2 recruitment to E1. EMBO J. 24, 439-451 (2005).

26. Walden, H. et al. The structure of the APPBP1-UBA3-NEDD8-ATP complex reveals the basis for selective ubiquitin-like protein activation by an E1. Mol. Cell 12, 1427-1437 (2003).

27. Lv, Z., Williams, K. M., Yuan, L., Atkison, J. H. \& Olsen, S. K. Crystal structure of a human ubiquitin E1-ubiquitin complex reveals conserved functional elements essential for activity. J. Biol. Chem. 293, 18337-18352 (2018).

28. Szczepanowski, R. H., Filipek, R. \& Bochtler, M. Crystal structure of a fragment of mouse ubiquitin-activating enzyme. J. Biol. Chem. 280, 22006-22011 (2005).

29. Huang, D. T. et al. Structural basis for recruitment of Ubc12 by an E2 binding domain in NEDD8's E1. Mol. Cell 17, 341-350 (2005).

30. Huang, D. T. et al. Basis for a ubiquitin-like protein thioester switch toggling E1-E2 affinity. Nature 445, 394-398 (2007).

31. Tokgoz, Z. et al. E1-E2 interactions in ubiquitin and Nedd8 ligation pathways. J. Biol. Chem. 287, 311-321 (2012).

32. Olsen, S. K. \& Lima, C. D. Structure of a ubiquitin E1-E2 complex: insights to E1-E2 thioester transfer. Mol. Cell 49, 884-896 (2013).

33. Lv, Z. et al. S. pombe Ubal-Ubc15 structure reveals a novel regulatory mechanism of ubiquitin E2 activity. Mol. Cell 65, 699-714 e6 (2017).

34. Seol, J. H. et al. Cdc53/cullin and the essential Hrt1 RING-H2 subunit of SCF define a ubiquitin ligase module that activates the E2 enzyme Cdc34. Genes Dev. 13, 1614-1626 (1999).

35. Kamura, T. et al. Rbx1, a component of the VHL tumor suppressor complex and SCF ubiquitin ligase. Science 284, 657-661 (1999).

36. Schulman, B. A. et al. Insights into SCF ubiquitin ligases from the structure of the Skp1-Skp2 complex. Nature 408, 381-386 (2000).

37. Zheng, N. et al. Structure of the Cul1-Rbx1-Skp1-F boxSkp2 SCF ubiquitin ligase complex. Nature 416, 703-709 (2002).

38. Hamilton, K. S. et al. Structure of a conjugating enzyme-ubiquitin thiolester intermediate reveals a novel role for the ubiquitin tail. Structure 9, 897-904 (2001).

39. Kamadurai, H. B. et al. Insights into ubiquitin transfer cascades from a structure of a UbcH5B ubiquitin-HECTNEDD4L complex. Mol. Cell 36, 1095-1102 (2009).

40. Page, R. C., Pruneda, J. N., Amick, J., Klevit, R. E. \& Misra, S. Structural insights into the conformation and oligomerization of E2 ubiquitin conjugates. Biochemistry 51, 4175-4187 (2012).

41. Wiener, R., Zhang, X., Wang, T. \& Wolberger, C. The mechanism of OTUB1mediated inhibition of ubiquitination. Nature 483, 618-622 (2012).

42. Middleton, A. J. \& Day, C. L. The molecular basis of lysine 48 ubiquitin chain synthesis by Ube2K. Sci. Rep. 5, 16793 (2015).

43. Pruneda, J. N. et al. Structure of an E3:E2 Ub complex reveals an allosteric mechanism shared among RING/U-box ligases. Mol. Cell 47, 933-942 (2012).

44. Dou, H., Buetow, L., Sibbet, G. J., Cameron, K. \& Huang, D. T. BIRC7-E2 ubiquitin conjugate structure reveals the mechanism of ubiquitin transfer by a RING dimer. Nat. Struct. Mol. Biol. 19, 876-883 (2012).

45. Plechanovová, A., Jaffray, E. G., Tatham, M. H., Naismith, J. H. \& Hay, R. T. Structure of a RING E3 ligase and ubiquitin-loaded E2 primed for catalysis. Nature 489, 115-120 (2012).

46. Kleiger, G., Saha, A., Lewis, S., Kuhlman, B. \& Deshaies, R. J. Rapid E2-E3 assembly and disassembly enable processive ubiquitylation of cullin-RING ubiquitin ligase substrates. Cell 139, 957-968 (2009).

47. Kleiger, G., Hao, B., Mohl, D. A. \& Deshaies, R. J. The acidic tail of the Cdc34 ubiquitin-conjugating enzyme functions in both binding to and catalysis with ubiquitin ligase SCFCdc4. J. Biol. Chem. 284, 36012-36023 (2009).

48. Ptak, C. et al. Functional and physical characterization of the cell cycle ubiquitin-conjugating enzyme CDC34 (UBC3). Identification of a functional determinant within the tail that facilitates CDC34 self-association. J. Biol. Chem. 269, 26539-26545 (1994).

49. Spratt, D. E. \& Shaw, G. S. Association of the disordered C-terminus of CDC34 with a catalytically bound ubiquitin. J. Mol. Biol. 407, 425-438 (2011).

50. Choi, Y. -S. et al. The human Cdc34 carboxyl terminus contains a noncovalent ubiquitin binding activity that contributes to SCF-dependent ubiquitination. J. Biol. Chem. 285, 17754-17762 (2010).

51. Huang, H. et al. E2 enzyme inhibition by stabilization of a low-affinity interface with ubiquitin. Nat. Chem. Biol. 10, 156-163 (2014).
52. Lee, I. \& Schindelin, H. Structural insights into E1-catalyzed ubiquitin activation and transfer to conjugating enzymes. Cell 134, 268-278 (2008).

53. Lv, Z. et al. Molecular mechanism of a covalent allosteric inhibitor of SUMO E1 activating enzyme. Nat. Commun. 9, 5145 (2018).

54. Pitluk, Z. W., McDonough, M., Sangan, P. \& Gonda, D. K. Novel CDC34 (UBC3) ubiquitin-conjugating enzyme mutants obtained by charge-to-alanine scanning mutagenesis. Mol. Cell. Biol. 15, 1210-1219 (1995).

55. Kolman, C. J., Toth, J. \& Gonda, D. K. Identification of a portable determinant of cell cycle function within the carboxyl-terminal domain of the yeast CDC34 (UBC3) ubiquitin conjugating (E2) enzyme. EMBO J. 11, 3081-3090 (1992).

56. Sandoval, D. et al. Ubiquitin-conjugating enzyme Cdc34 and ubiquitin ligase Skp1-cullin-F-box ligase (SCF) interact through multiple conformations. $J$. Biol. Chem. 290, 1106-1118 (2015).

57. Wu, K., Chen, A., Tan, P. \& Pan, Z. -Q. The Nedd8-conjugated ROC1-CUL1 core ubiquitin ligase utilizes Nedd8 charged surface residues for efficient polyubiquitin chain assembly catalyzed by Cdc34. J. Biol. Chem. 277, 516-527 (2002).

58. Yuan, L., Lv, Z., Atkison, J. H. \& Olsen, S. K. Structural insights into the mechanism and E2 specificity of the RBR E3 ubiquitin ligase HHARI. Nat. Commun. 8, 211 (2017).

59. Patel, A., Sibbet, G. J. \& Huang, D. T. Structural insights into non-covalent ubiquitin activation of the cIAP1-UbcH5B ubiquitin complex. J. Biol. Chem. 294, 1240-1249 (2019).

60. Wickliffe, K. E., Lorenz, S., Wemmer, D. E., Kuriyan, J. \& Rape, M. The mechanism of linkage-specific ubiquitin chain elongation by a single-subunit E2. Cell 144, 769-781 (2011).

61. Suryadinata, R. et al. Molecular and structural insight into lysine selection on substrate and ubiquitin lysine 48 by the ubiquitin-conjugating enzyme Cdc 34 . Cell Cycle 12, 1732-1744 (2013).

62. Petroski, M. D. \& Deshaies, R. J. Mechanism of lysine 48-linked ubiquitinchain synthesis by the cullin-RING ubiquitin-ligase complex SCF-Cdc34. Cell 123, 1107-1120 (2005).

63. Saha, A., Lewis, S., Kleiger, G., Kuhlman, B. \& Deshaies, R. J. Essential role for ubiquitin-ubiquitin-conjugating enzyme interaction in ubiquitin discharge from Cdc34 to substrate. Mol. Cell 42, 75-83 (2011).

64. Hill, S., Harrison, J. S., Lewis, S. M., Kuhlman, B. \& Kleiger, G. Mechanism of lysine 48 selectivity during polyubiquitin chain formation by the Ube2R1/2 ubiquitin-conjugating enzyme. Mol. Cell. Biol. 36, 1720-1732 (2016).

65. Schwob, E., Böhm, T., Mendenhall, M. D. \& Nasmyth, K. The B-type cyclin kinase inhibitor p40SIC1 controls the G1 to S transition in S. cerevisiae. Cell 79, 233-244 (1994)

66. Butz, N. et al. The human ubiquitin-conjugating enzyme Cdc34 controls cellular proliferation through regulation of p27Kip1 protein levels. Exp. Cell Res 303, 482-493 (2005).

67. Adams, P. D. et al. PHENIX: a comprehensive Python-based system for macromolecular structure solution. Acta Crystallogr. Sect. D: Biol. Crystallogr. 66, 213-221 (2010)

68. Emsley, P. \& Cowtan, K. Coot: model-building tools for molecular graphics. Acta Crystallogr. Sect. D: Biol. Crystallogr. 60, 2126-2132 (2004).

69. Williams, C. J. et al. MolProbity: more and better reference data for improved all-atom structure validation. Protein Sci. 27, 293-315 (2018).

\section{Acknowledgements}

We thank Megan Sheridan for assisting in crystallization of Cdc34 apo, and Lingmin Yuan, Christopher Davies, and Miklos Bekes for critically reading the manuscript. X-ray diffraction data were collected at SER-CAT 22-ID and NE-CAT 24-ID-E beamlines at the Advanced Photon Source, Argonne National Laboratory. This work is based upon research conducted at the Northeastern Collaborative Access Team beamlines, which are funded by the National Institute of General Medical Sciences from the National Institutes of Health (P41 GM103403). The Pilatus $6 \mathrm{M}$ detector on 24-ID-C beamline is funded by a NIH-ORIP HEI grant (S10 RR029205). This research used resources of the Advanced Photon Source, a U.S. Department of Energy (DOE) Office of Science User Facility operated for the DOE Office of Science by Argonne National Laboratory under Contract No. DE-AC02-06CH11357. The X-ray crystallography facility used for this work is supported by the Office of the Vice President for Research at the Medical University of South Carolina. The liquid handling robot used was purchased via an NIH Shared Instrumentation Award (S10 RR027139-01). Research reported in this publication was supported by NIH R01 GM115568 and R01 GM128731 (S.K.O.), and NIH P01 CA098101 and R01 CA093237 (J.A.D.). This work was also supported, in part, by NIH F30 CA216921 (K.M.W.), the Abney Foundation (K.M.W.), NIH T32 CA193201 (J.H.A.), and NIH T32 DE017551 (S.Q.). The content of this study is solely the responsibility of the authors and does not necessarily represent the official views of the NIH. 


\section{Author contributions}

Structural experiments including crystallization, X-ray data collection/processing, model building/refinement, and structural analyses were performed by K.M.W., J.H.A. and S.K. O. K.M.W. and S.S.A. conducted biochemical assays. S.Q. and J.A.D. designed and conducted mammalian cell assays. The manuscript was written by K.M.W. and S.K.O

\section{Additional information}

Supplementary Information accompanies this paper at https://doi.org/10.1038/s41467019-11061-8.

Competing interests: The authors declare no competing interests.

Reprints and permission information is available online at http://npg.nature.com/ reprintsandpermissions/

Peer review information: Nature Communications thanks the anonymous reviewers for their contribution to the peer review of this work. Peer reviewer reports are available.
Publisher's note: Springer Nature remains neutral with regard to jurisdictional claims in published maps and institutional affiliations.

(c) (i) Open Access This article is licensed under a Creative Commons Attribution 4.0 International License, which permits use, sharing, adaptation, distribution and reproduction in any medium or format, as long as you give appropriate credit to the original author(s) and the source, provide a link to the Creative Commons license, and indicate if changes were made. The images or other third party material in this article are included in the article's Creative Commons license, unless indicated otherwise in a credit line to the material. If material is not included in the article's Creative Commons license and your intended use is not permitted by statutory regulation or exceeds the permitted use, you will need to obtain permission directly from the copyright holder. To view a copy of this license, visit http://creativecommons.org/ licenses/by/4.0/.

(C) The Author(s) 2019 